Henning Dathe

Die Halbwinkelsubstitution und ihre Anwendungen

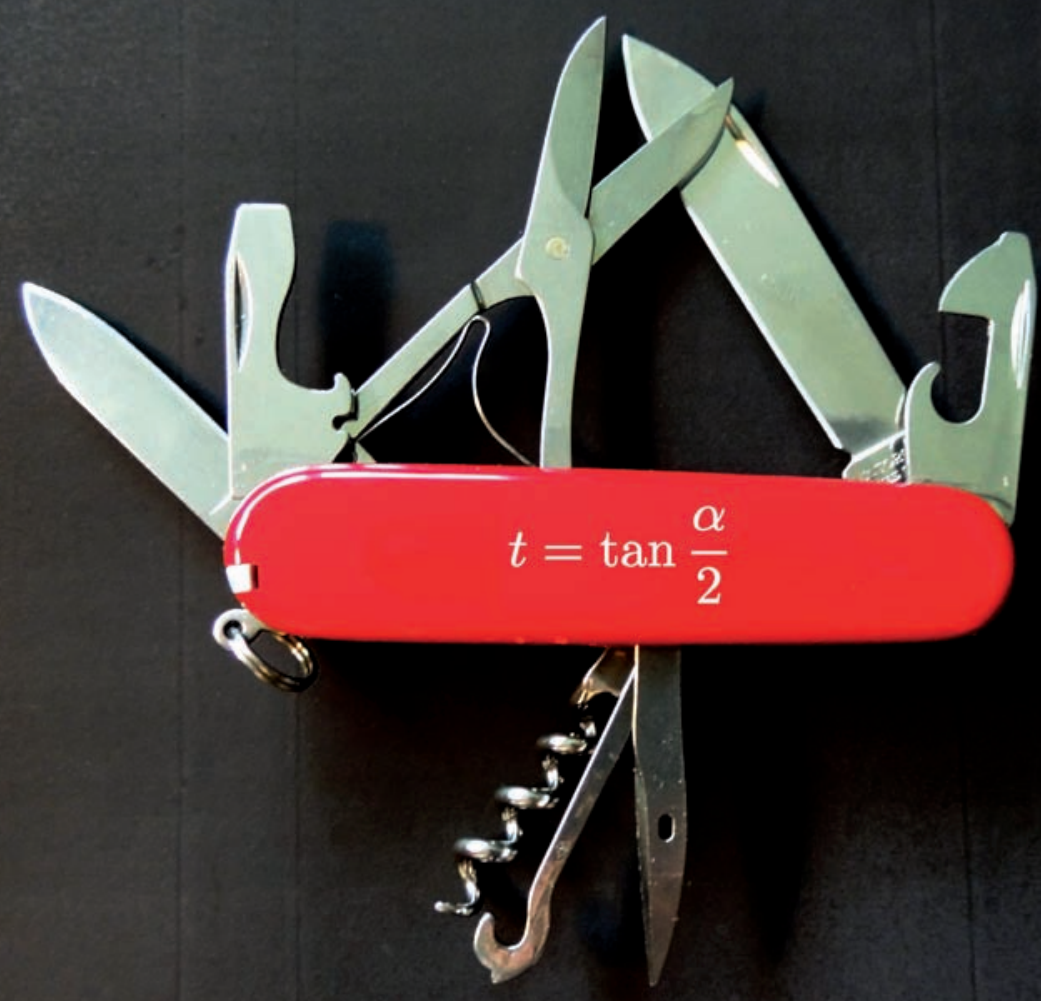

Universitätsdrucke Göttingen 

Henning Dathe

Die Halbwinkelsubstitution und ihre Anwendungen

Dieses Werk ist lizenziert unter einer

Creative Commons

Namensnennung - Weitergabe unter gleichen Bedingungen

4.0 International Lizenz.

(ㄷ) (1) ( $)$ 
erschienen in der Reihe der Universitätsdrucke im Universitätsverlag Göttingen 2015 
Henning Dathe

\section{Die Halbwinkelsubstitution und ihre Anwendungen}

Forschungsbericht

aus der Arbeitsgruppe Biomechanik

Abteilung Kieferorthopädie

Universitätsmedizin Göttingen

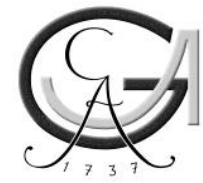

Universitätsverlag Göttingen 2015 


\section{Bibliographische Information der Deutschen Nationalbibliothek}

Die Deutsche Nationalbibliothek verzeichnet diese Publikation in der Deutschen Nationalbibliographie; detaillierte bibliographische Daten sind im Internet über $<$ http://dnb.dnb.de $>$ abrufbar.

Anschrift des Autors

Henning Dathe

E-Mail: hdathe1@gwdg.de

Dieses Buch ist auch als freie Onlineversion über die Homepage des Verlags sowie über den Göttinger Universitätskatalog (GUK) bei der Niedersächsischen Staats- und Universitätsbibliothek Göttingen (http://www.sub.uni-goettingen.de) erreichbar. Es gelten die Lizenzbestimmungen der Onlineversion.

Satz und Layout: Henning Dathe Umschlaggestaltung: Jutta Pabst Titelabbildung: Henning Dathe

(C) 2015 Universitätsverlag Göttingen http://univerlag.uni-goettingen.de ISBN: 978-3-86395-215-0 


\section{Inhaltsverzeichnis}

$\begin{array}{lll}1 & \text { Einleitung } & 7\end{array}$

1.1 Elementare Herleitung . . . . . . . . . . . . . . . . 9 9

$1.2 \quad$ Ein Dreieck als Merkregel _. . . . . . . . . . . . . . . . . 11

1.3 Geometrische Veranschaulichung . . . . . . . . . . . . 12

1.4 Ein Austlug ins Komplexe . . . . . . . . . . . . . . . . 13

1.5 Etwas Computeralgebra . . . . . . . . . . . . . . 16

$\square .5$. Mathematica . . . . . . . . . . . . . . 16

$\llbracket .5 .2$ Maxima . . . . . . . . . . . . . . . 16

ए.5.3 Reduce . . . . . . . . . . . . . . . 17

$\square .5 .4$ Axiom . . . . . . . . . . . . . . . . 17

1.6 Kleine Argumente $\ldots \ldots \ldots \ldots \ldots$

2 Anwendungen in der reinen Mathematik 21

2.1 Integration rationaler Funktionen . . . . . . . . . . . . . 21

2.2 Pythagoreische Zahlentripel . . . . . . . . . . . . . . . . 22

2.3 Kosinussatz und Halbwinkelsatg . . . . . . . . . . . . 23

2.4 Höhere Vielfache . . . . . . . . . . . . . . . . . . . . 25

2.4.] Die Koeflizienten der höheren Vielfachen . . . . . . 26

2.5 Die Einheitswurzeln . . . . . . . . . . . . . . . 27

2.6 Eine stereografische Projektion $\ldots \ldots \ldots \ldots$

2.6 .1 In drei Dimensionen . . . . . . . . . . . . . 31

2.7 Inversion von Polarkoordinaten . . . . . . . . . . . 31

2.8 Inversion von Kugelkoordinaten . . . . . . . . . . . . . . 32

2.9 Eine Verallgemeinerung auf zwei Argumente . . . . . . . . 33

2.10 Die erste Eisenstein-Finktion . . . . . . . . . . . 34

2.11 Eine Differentialgleichung vom Riccati-Typ . . . . . . 36

$\begin{array}{lll}3 & \text { Drehungen und Kinematik } & 39\end{array}$

3.1 Ebene Drehungen . . . . . . . . . . . . . . . . . . . . . . . 39

3.1.1 Rückgewinnung des Parameters . . . . . . . . . . . 39

3.1.2 Zusammensetzung von ebenen Drehungen . . . . . 40

3.1 .3 Eine Parametrisierung mit Nebenbedingungen . . 40 
3.1.4 Eine Matrizenschar . . . . . . . . . . . . . . . 41

3.1.5 Die direkte Ableitung einer Drehmatrix . . . . . . 41

3.1.6 Die Darstellung im Vierteltangens . . . . . . . . . 42

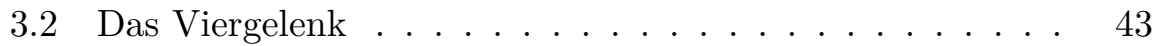

3.3 Räumliche Drehungen . . . . . . . . . . . . . . . . . . 45

3.3.1 Rückgewinnung der Rodrigues-Parameter . . . . . 46

3.3.2 Hintereinanderschaltung von 3D-Drehungen . . . . 46

3.3 .3 Die Cayleyschen Formeln . . . . . . . . . . . . . 47

3.3.4 Eulerparameter . . . . . . . . . . . . . . . . . . . 48

3.3.5 Die Ableitung einer Drehmatrix . . . . . . . . . 50

3.4 Drehungen als komplexe Zahlen und Matrizen . . . . . . . 51

3.5 Instantane Kinematik . . . . . . . . . . . 52

4 Anwendungen in der Physik 55

4.1 Verteilungsfunktionen . . . . . . . . . . . . . . 55

$4.2 \quad$ Die Besetzungszahlditferenz . . . . . . . . . . . . 56

4.3 Die Bloch-Gleichung . . . . . . . . . . . . . . . . . . . . . . . 57

4.3 .1 Freie Präzession . . . . . . . . . . . . 57

$4.3 .2 \quad$ Mit Dämpfung (Relaxation) . . . . . . . . . . . . 58

4.3 .3 Erzwungene Schwingungen . . . . . . . . . . . 58

4.3 .4 Stationäre Lösungen . . . . . . . . . . . . . . . . . 59

4.3 .5 Die Riccati-Gleichung und Pulse . . . . . . . . . 59

4.4 Die Flash-Sequenz . . . . . . . . . . . . . . . . . . . . . . . . 60

4.5 Die balancierte SSFP-Sequenz . . . . . . . . . . . . . . . 63

4.6 Die unbalancierte SSFP-Sequenz . . . . . . . . . . . . . . 65

4.7 Sequenzen aus Einzelpulsen $\ldots \ldots \ldots \ldots$. . . . . . . 67 


\section{Einleitung}

Die Halbwinkelsubstitution ist aus den Anfängervorlesungen in Mathematik als wesentlicher Rechentrick bekannt bei der Integration rationaler Funktionen der Winkelfunktionen $\cos (\alpha)$ und $\sin (\alpha)$. So schreibt etwa SPIVAK in seinem Lehrbuch zur Analysis ([Spi80], pp. 360-361)

"The world's sneakiest ${ }^{\mathbb{m}}$ substitution is undoubtedly

$$
\begin{array}{r}
t=\tan \frac{x}{2}, \\
x=2 \arctan t, d x=\frac{2}{1+t^{2}} d t . "
\end{array}
$$

Das Buch benötigt Vorkenntnisse aus der Analysis, linearen Algebra und den Differentialgleichungen; ein wenig Funktionentheorie ist nützlich. Eine Vorlesung über mathematische Methoden der Physik vertieft dabei die Zusammenhänge im Hinblick auf die Anwendungen, gerade was Vektor- und Tensorrechnung anbelangt. Das letzte Kapitel behandelt vorwiegend Beispiele aus der Kernspintomografie. Es bringt daher für Leser mit entsprechenden Vorkenntnissen am meisten Gewinn. Nach etwa dem dritten oder vierten Semester in Physik, Mathematik oder Ingenieurswissenschaften sollte das Buch aber verständlich sein.

Die Substitution besitzt eine geometrische Veranschaulichung, da sie eine stereografische Projektion der Zahlengerade darstellt. Im Raum entspricht sie dem Betrag der stereografischen Projektion. Daher ist sie sinnvoll, sobald eine Kreis- oder Kugelsymmetrie vorliegt. Die Halbwinkelsubstitution algebraisiert Winkelfunktionen, und ist daher über ihre ursprüngliche Bedeutung hinaus nützlich in allen Problemen, in denen solche Funktionen vorkommen. Beispiele sind die Inversion von Polarkordinaten oder die Gewinnung rationaler Darstellungen von Drehmatrizen. Die Transformation führt direkt auf Anwendungen in Mathematik, Kinematik des starren Körpers uva. bis hin zur Zahlentheorie. Es existiert

\footnotetext{
${ }^{1}$ Übersetzt etwa: „raffiniert“ mit Nebenbedeutung „hinterhältig“. Ich verdanke den Hinweis auf dieses Zitat der englischsprachigen Wikipedia.
} 


\section{Einleitung}

eine Beziehung zu einer speziellen nichtlinearen Differentialgleichung, der RICCATI-Gleichung.

Ferner algebraisiert die im Komplexen verwandte Transformation

$$
h=\tanh \frac{x}{2}
$$

hyperbolische und Exponentialfunktionen. Da solche Funktionen als Lösungen von in Physik und Technik wichtigen linearen Differentialgleichungen auftauchen, sind die Substitutionen in vielen praxisrelevanten Fällen nützlich.

Die Halbwinkelsubstitution und ihr hyperbolisches Analogon machen viele wichtige derartige Probleme der Computeralgebra zugänglich. Sie schaffen so eine Zone von exakten Lösungen, die bislang wegen des immensen Rechenaufwandes verborgen oder unbearbeitet blieben. Daher zieht sich Computeralgebra wie ein roter Faden durch diese Arbeit.

Beginnend mit elementaren Überlegungen wendet sich das Buch zunächst der reinen Mathematik zu (sofern mir das als Physiker überhaupt zusteht). Danach wird spezieller auf Drehungen und Kinematik in Ebene und Raum eingegangen. Am Schluss folgen diejeningen Anwendungen in der Physik, insbesondere der Kernspintomografie, die überhaupt erst zur Beschäftigung mit der Materie führten. Die Begriffswelt der Kernspintomografie ist sehr speziell und umfangreich. Ich habe mich bemüht, die korrekten Termini zu benutzen und verweise den interessierten Leser auf das im Internet zugängliche MR-Glossar der Firma Siemens.

Man beendet kein Buch, sondern verlässt es nur. Es gäbe den zusammengetragenen Beispielen sicher noch einige hinzuzufügen. Von einem Anspruch auf Vollständigkeit muss dieses Buch daher weit entfernt bleiben. Die Methode der Halbwinkelsubstitution ist eben doch zu allgemein, um erschöpfend in Beispielen dargestellt zu werden. Mir bleibt die Hoffnung, dass es wenigstens einen Leser gebe, den diese Zusammenstellung erhellt.

Ich bedanke mich insbesondere bei meinem Studienfreund GunTHER HeLms, welcher mich auf dieses interessante Thema brachte und der diese Arbeit an zahlreichen Stellen zu verbessern half. 


\subsection{Elementare Herleitung}

\subsection{Elementare Herleitung}

Durch elementargeometrische Überlegungen lassen sich die Additionstheoreme für die Winkelfunktionen herleiten. Sie lauten

$$
\begin{aligned}
& \sin (\alpha \pm \beta)=\sin \alpha \cos \beta \pm \cos \alpha \sin \beta \\
& \cos (\alpha \pm \beta)=\cos \alpha \cos \beta \mp \sin \alpha \sin \beta .
\end{aligned}
$$

Aus diesen Formeln gewinnt man die Formeln für die doppelten Winkel

$$
\sin 2 \varphi=2 \sin \varphi \cos \varphi
$$

und

$$
\cos 2 \varphi=\cos ^{2} \varphi-\sin ^{2} \varphi=1-2 \sin ^{2} \varphi=2 \cos ^{2} \varphi-1 .
$$

Aus diesen nun folgen zwei Formeln für den Tangens des ursprünglichen Winkels, nämlich

$$
\begin{aligned}
\frac{\sin 2 \varphi}{1+\cos 2 \varphi} & =\frac{2 \sin \varphi \cos \varphi}{1+\left(1-2 \sin ^{2} \varphi\right)}=\frac{2 \sin \varphi \cos \varphi}{2-2 \sin ^{2} \varphi}=\frac{\sin \varphi \cos \varphi}{\cos ^{2} \varphi} \\
& =\frac{\sin \varphi}{\cos \varphi}=\tan \varphi
\end{aligned}
$$

und (man lese beide Formelketten zum Beweis auch von rechts nach links)

$$
\frac{1-\cos 2 \varphi}{\sin 2 \varphi}=\frac{1-\left(1-\sin ^{2} \varphi\right)}{2 \sin \varphi \cos \varphi}=\frac{2 \sin ^{2} \varphi}{2 \sin \varphi \cos \varphi}=\frac{\sin \varphi}{\cos \varphi}=\tan \varphi .
$$

Analog lassen sich die Winkelfunktionen des doppelten Winkels durch den Tangens des ursprünglichen Winkels ausdrücken, also

$$
\frac{2 \tan \varphi}{1+\tan ^{2} \varphi}=\frac{2 \sin \varphi / \cos \varphi}{1+\sin ^{2} \varphi / \cos ^{2} \varphi}=\frac{2 \sin \varphi \cos \varphi}{\cos ^{2} \varphi+\sin ^{2} \varphi}=\sin 2 \varphi
$$

und (wieder auch von rechts nach links zu lesen)

$$
\frac{1-\tan ^{2} \varphi}{1+\tan ^{2} \varphi}=\frac{1-\sin ^{2} \varphi / \cos ^{2} \varphi}{1+\sin ^{2} \varphi / \cos ^{2} \varphi}=\frac{\cos ^{2} \varphi-\sin ^{2} \varphi}{\cos ^{2} \varphi+\sin ^{2} \varphi}=\cos 2 \varphi .
$$

Setzt man nun $\varphi=: \frac{\alpha}{2}$ und $t:=\tan \frac{\alpha}{2}$, so lauten die eben erhaltenen Gleichungen

$$
t=\frac{\sin \alpha}{1+\cos \alpha}=\frac{1-\cos \alpha}{\sin \alpha}
$$




\section{Einleitung}

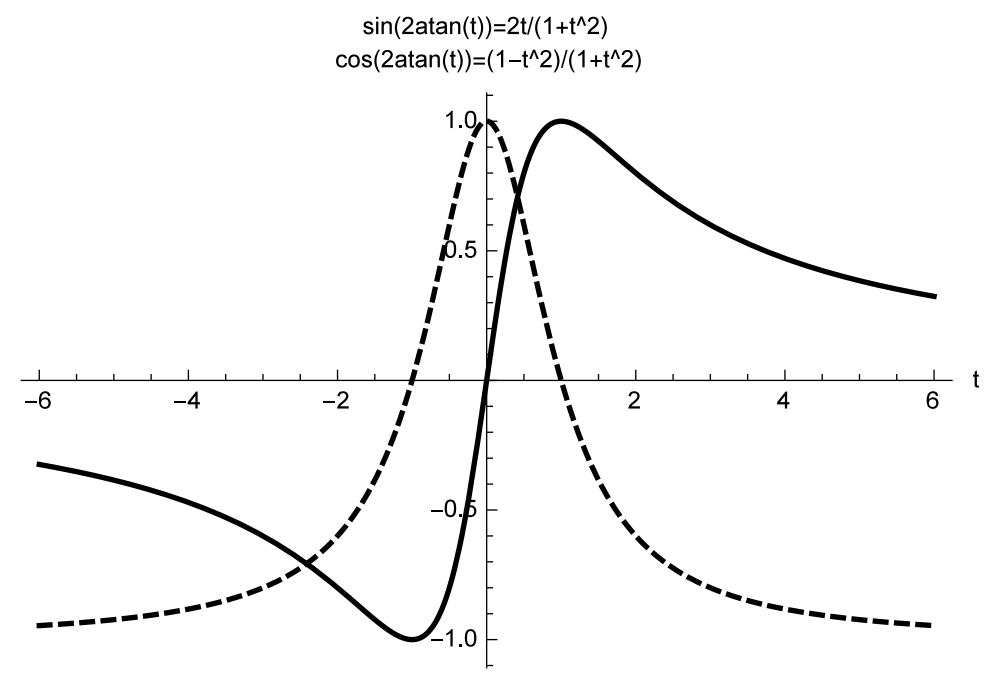

Abbildung 1.1: Die Funktionen $\sin \alpha=\frac{2 t}{1+t^{2}}$ und $\cos \alpha=\frac{1-t^{2}}{1+t^{2}}$ als Funktion des Tangens des halben Winkels. Der Definitionsbereich $\alpha \in(-\pi, \pi)$ wird abgebildet auf $t \in(-\infty, \infty)$. Genauer bekommt man für jeden Vollwinkelbereich $\alpha \neq n \pi$, wobei $\mathrm{n}$ eine ganze Zahl ist, wieder den gesamten Bereich für $t$. Der Wertebereich bleibt natürlich ungeändert.

sowie

$$
\sin \alpha=\frac{2 t}{1+t^{2}} \quad \text { und } \quad \cos \alpha=\frac{1-t^{2}}{1+t^{2}} .
$$

Die Graphen dieser Funktionen sind in Fig. $\square$ d dargestellt.

Die Quadrate der Winkelfunktionen lassen sich ineinander umrechnen. Mit den Abkürzungen $s:=\sin \left(\frac{\alpha}{2}\right)$ und $c:=\cos \left(\frac{\alpha}{2}\right)$ hat man zusätzlich

$$
\sin ^{2}\left(\frac{\alpha}{2}\right)=\frac{s^{2}}{s^{2}+c^{2}}=\frac{s^{2} / c^{2}}{s^{2} / c^{2}+1}=\frac{t^{2}}{1+t^{2}}
$$

und

$$
\cos ^{2}\left(\frac{\alpha}{2}\right)=1-s^{2}=1-\frac{t^{2}}{1+t^{2}}=\frac{1+t^{2}-t^{2}}{1+t^{2}}=\frac{1}{1+t^{2}} .
$$

Bemerkenswerterweise haben diese Ausdrücke denselben Nenner wie die aus (미). 


\subsection{Ein Dreieck als Merkregel}

In Abb. ए.2 ist ein rechtwinkliges Dreieck abgebildet, denn: wie man unschwer nachrechnet gilt der Satz des Pythagoras

$$
\left(1-t^{2}\right)^{2}+(2 t)^{2}=1-2 t^{2}+t^{4}+4 t^{2}=1+2 t^{2}+t^{4}=\left(1+t^{2}\right)^{2} .
$$

Die Winkelfunktionen an diesem Dreieck ergeben sich damit aus den

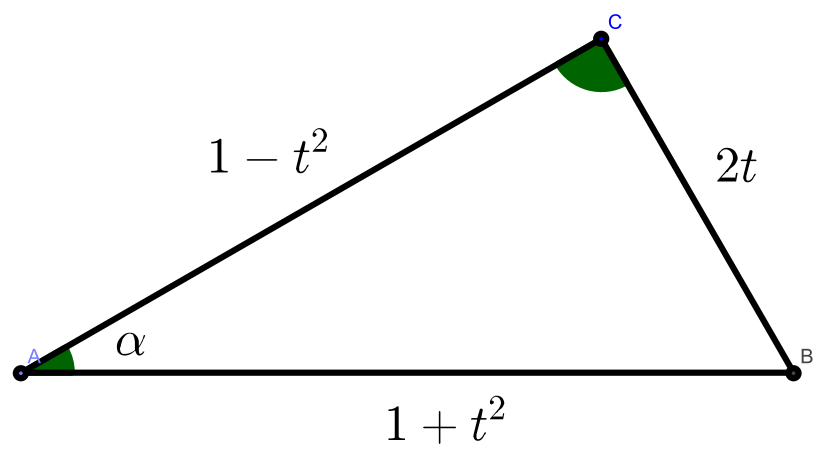

Abbildung 1.2: Die Merkregel zur Halbwinkelsubstitution.

Definitionen

$$
\begin{aligned}
\sin \alpha & =\frac{\text { Gegenkathete }}{\text { Hypothenuse }}=\frac{2 t}{1+t^{2}} \\
\cos \alpha & =\frac{\text { Ankathete }}{\text { Hypothenuse }}=\frac{1-t^{2}}{1+t^{2}} \\
\tan \alpha & =\frac{\text { Gegenkathete }}{\text { Ankathete }}=\frac{2 t}{1-t^{2}}
\end{aligned}
$$

Das sind genau die Formeln (따), und mit (ㄸ. $)$ erhält man die Bedeutung von $t$ zurück. 


\section{Einleitung}

\subsection{Geometrische Veranschaulichung}

Die Konstruktion der Abb. [.3 beginnt mit dem Einheitskreis um den Ursprung $O$. Er enthalte die Punkte $A=(-1,0), D=(1,0)$ und den beliebigen Punkt $C$.

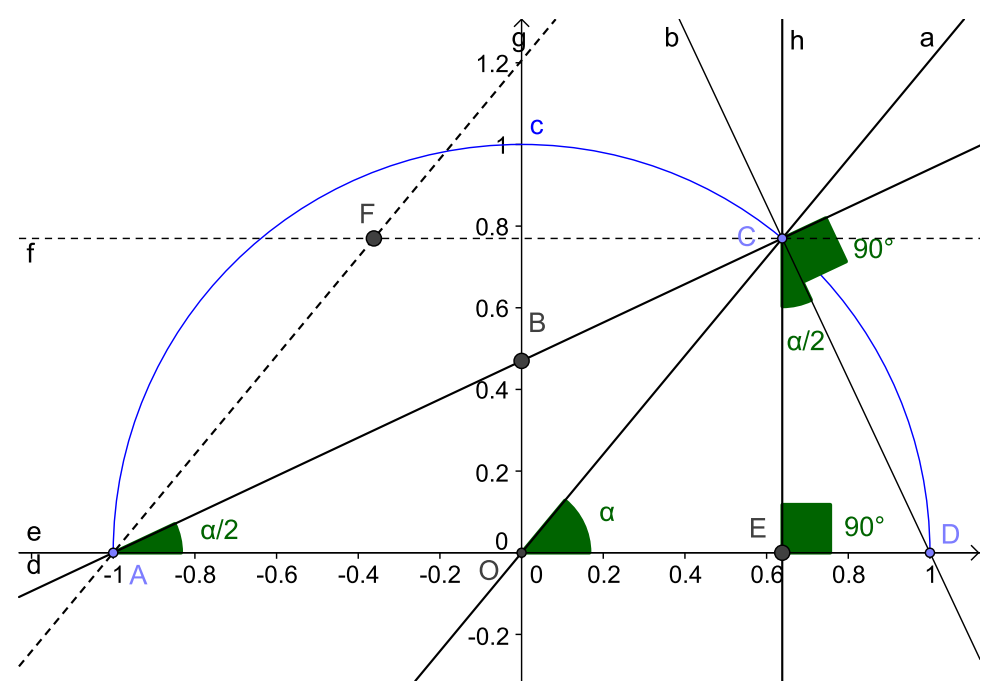

Abbildung 1.3: Die Visualisierung der Halbwinkelsubstitution. Weitere Einzelheiten im Text des Abschnittes [..3.

Ergänzt man $\triangle(A O C)$ mit den Seiten $\overline{A O}=1=\overline{O C}$ um den Punkt $F$ zur Raute (gestrichelte Linien), so ist die Strecke $A C$ ihre Winkelhalbierende. Daher ist $\angle(O A C)=\angle(E O C) / 2$.

$\triangle(O E C)$ : Wegen $\overline{O C}=1$ ist $\overline{E C}=\sin \alpha$ und $\overline{O E}=\cos \alpha$.

$\triangle(A O B)$ : Wegen $\overline{A O}=1$ ist $t:=\overline{O B}=\tan \alpha / 2$.

$\triangle(A E C)$ :

$$
\tan \alpha / 2=\frac{\overline{E C}}{\overline{A E}}=\frac{\overline{E C}}{\overline{A O}+\overline{O E}}=\frac{\sin \alpha}{1+\cos \alpha} .
$$

$\triangle(C E D)$ : Da $\angle(A C D)$ und $\angle(C E A)$ beides rechte Winkel sind, ist auch $\angle(E C D)=\angle(E A C)=\tan \alpha / 2$ und es gilt

$$
\tan \alpha / 2=\frac{\overline{E D}}{\overline{E C}}=\frac{\overline{O D}-\overline{O E}}{\overline{E C}}=\frac{1-\cos \alpha}{\sin \alpha} .
$$

Damit sind die Formeln (ㅁ. $)$ veranschaulicht. 
$\triangle(A O B):$ Da $\angle(B O A)$ ein rechter Winkel ist gilt $\overline{A B}=\sqrt{1+t^{2}}$. Quadrieren der Definitionsgleichungen liefert dann

$$
\sin ^{2}\left(\frac{\alpha}{2}\right)=\frac{t^{2}}{1+t^{2}} \quad \text { und } \quad \cos ^{2}\left(\frac{\alpha}{2}\right)=\frac{1}{1+t^{2}} \quad .
$$

Damit sind auch die Formeln (미) und(ㅁ.

\subsection{Ein Ausflug ins Komplexe}

Natürlich hätte man statt der elementaren Überlegungen am Anfang die Additionstheoreme auch mit der EulERschen Formel

$$
e^{i \varphi}=\cos \varphi+i \sin \varphi
$$

herleiten können. Durch Vergleich von Real- und Imaginärteil erhält man

$$
\sin \varphi=\frac{1}{2 i}\left(e^{i \varphi}-e^{-i \varphi}\right) \quad \text { und } \quad \cos \varphi=\frac{1}{2}\left(e^{i \varphi}+e^{-i \varphi}\right) \quad .
$$

Vergleicht man das mit den Definitionen für die hyperbolischen Funktionen

$$
\sinh \varphi=\frac{1}{2}\left(e^{\varphi}-e^{-\varphi}\right) \quad \text { und } \cosh \varphi=\frac{1}{2}\left(e^{\varphi}+e^{-\varphi}\right) \quad,
$$

so erhält man sofort

$$
\sinh i \varphi=\frac{1}{2}\left(e^{i \varphi}-e^{-i \varphi}\right)=i \frac{1}{2 i}\left(e^{i \varphi}-e^{-i \varphi}\right)=i \sin \varphi
$$

und

$$
\cosh i \varphi=\frac{1}{2}\left(e^{i \varphi}+e^{-i \varphi}\right)=\cos \varphi
$$

Folglich gilt auch $\tanh i \varphi=i \tan \varphi$. Daher gelten analog zu den Formeln (ㄷ.J) unter Einführung der Drehung $x:=i \varphi$ und

$$
h:=\tanh \left(\frac{x}{2}\right)=\tanh \left(\frac{i \varphi}{2}\right)=i \tan \left(\frac{\varphi}{2}\right)=i t
$$

die Gleichungen

$$
\sinh x=\sinh i \varphi=i \sin \varphi=i \frac{2 t}{1+t^{2}}=\frac{2 h}{1-h^{2}}
$$




\section{Einleitung}

und

$$
\cosh x=\cosh i \varphi=\cos \varphi=\frac{1-t^{2}}{1+t^{2}}=\frac{1+h^{2}}{1-h^{2}} .
$$

Diese neue Substitution des halben Arguments („Winkel“ wäre hier unangebracht) algebraisiert also rationale Funktionen der hyperbolischen Funktionen. Der Wertebereich des hyperbolischen Tangens $h$, also der Definitionsbereich des hyperbolischen Arkustangens, liegt im Bereich $(-1,1)$.

In Computeralgebra, hier Mathematica, kann diese Substitution etwa realisiert werden durch

\section{Simplify[TrigExpand[Sinh $[\mathrm{x}] / . \mathrm{x} \rightarrow 2 \operatorname{ArcTanh}[\mathrm{h}]]]$ \\ Simplify [TrigExpand [Cosh $[\mathrm{x}] / . \mathrm{x} \rightarrow 2 \operatorname{ArcTanh}[\mathrm{h}]]]$}

wobei für $\sinh x$ oder $\cosh x$ die zu vereinfachende rationale Funktion einzusetzen ist.

Setzt man $E:=e^{x}$, so gilt wegen

$$
h=\tanh \frac{x}{2}=\frac{\sinh \frac{x}{2}}{\cosh \frac{x}{2}}=\frac{e^{\frac{x}{2}}-e^{-\frac{x}{2}}}{e^{\frac{x}{2}}+e^{-\frac{x}{2}}}=\frac{e^{x}-1}{e^{x}+1}=\frac{E-1}{E+1}
$$

Diese Funktion $h(E)$ ist invertierbar zu $E(h)$, und man erhält

$$
E=\frac{1+h}{1-h}
$$

Schneller sieht man das ein mit

$$
\begin{aligned}
\exp (x) & =\cosh (x)+\sinh (x)=\left(\frac{1+h^{2}}{1-h^{2}}+\frac{2 h}{1-h^{2}}\right) \\
& =\frac{(1+h)^{2}}{(1-h)(1+h)}=\frac{1+h}{1-h} .
\end{aligned}
$$

Die Substitution rationalisiert also gleichzeitig auch Exponentialterme des ursprünglichen Arguments. Das tut $E$ selbst zwar auch schon, aber diese beiden Substitutionen werden bei Problemen an der Grenze der Behandelbarkeit ein unterschiedliches Verhalten zeigen. Die Funktion (‥2.5) ist eine spezielle MöBıUs-Transformation der komplexen Ebene.

In Computeralgebra, hier wieder Mathematica, wird die Substitution durch die Befehlskette

Simplify[TrigExpand[ExpToTrig[Exp[2 ArcTanh[h]]]] 


\subsection{Ein Ausflug ins Komplexe}

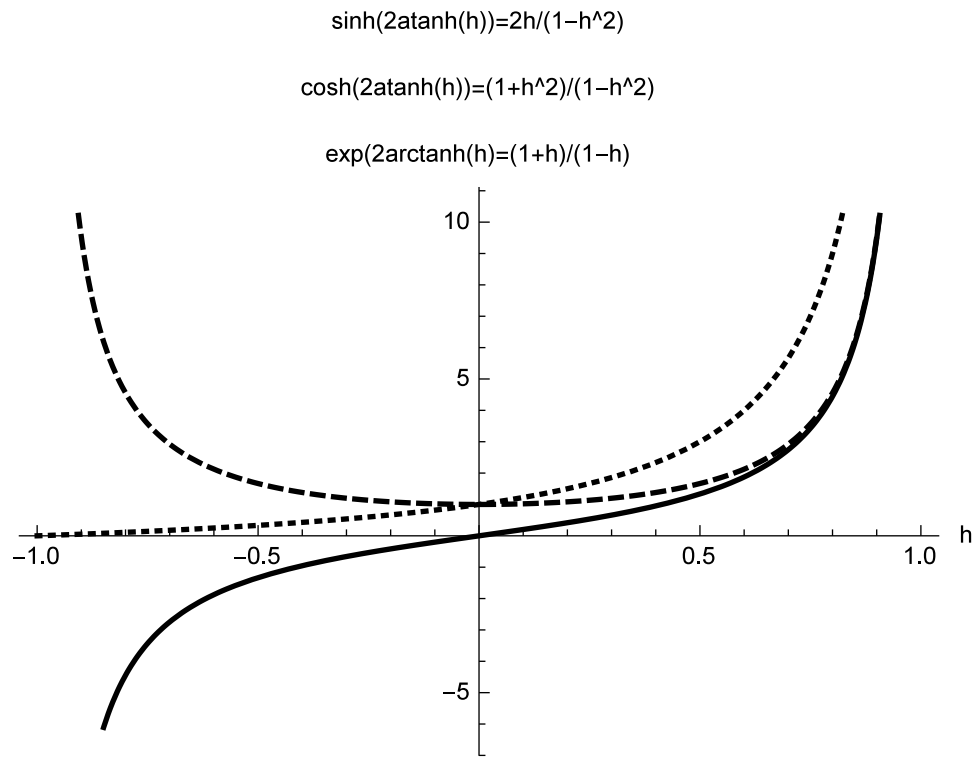

Abbildung 1.4: Die Funktionen $\sinh x=\frac{2 h}{1-h^{2}}$ und $\cosh x=\frac{1+h^{2}}{1-h^{2}}$ sowie $\exp x=\frac{1+h}{1-h}$ als Funktion des Tangenshyperbolikus des halben Arguments.

vollzogen.

Die Graphen dieser neuen Funktionen sind in Fig. 1.4 dargestellt.

Die Formel (ए.25) korrespondiert mit der logarithmischen Darstellung des hyperbolischen Arkustangens, denn es ist

$$
\operatorname{arctanh} h=\frac{x}{2}=\frac{1}{2} \ln E=\frac{1}{2} \ln \frac{1+h}{1-h} .
$$

Eine weitere Besonderheit ergibt sich bei Anwesenheit zweier oder mehrerer Exponentialfunktionen. Etwa aus

$$
e^{x}-e^{y}=e^{\frac{x+y}{2}}\left(e^{\frac{x-y}{2}}-e^{-\frac{x-y}{2}}\right)=e^{\frac{x+y}{2}} 2 \sinh \frac{x-y}{2}
$$

liest man ab, dass auch die halben Summen und Differenzen der Exponenten geeignete Kandidaten für die obige Substitution sind. 


\section{Einleitung}

\subsection{Etwas Computeralgebra}

Computeralgebra-Systeme (CAS) sind Programme zur automatisierten symbolischen Manipulation algebraischer Ausdrücke. Sie sind besonders vorteilhaft bei langen Ausdrücken, die per Hand zu mühsam zu bearbeiten sind.

In diesem Abschnitt sollen die elementaren Substitutionen für verschiedene Computeralgebra-Systeme vorgestellt werden. Generell werden die Ausdrücke erst expandiert und dann wieder vereinfacht.

Bis auf das kommerzielle Mathematica sind die vorgestellten CAS alle frei erhältlich.

\subsubsection{Mathematica}

Mit der Funktion

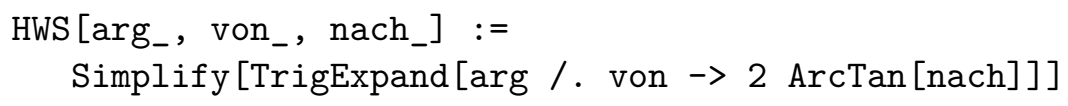

bekommt man die Funktionen sin, cos, tan und ihre Quadrate algebraisiert. Etwas schwerer ist das bei den hyperbolischen Funktionen. Hier hilft die Funktion

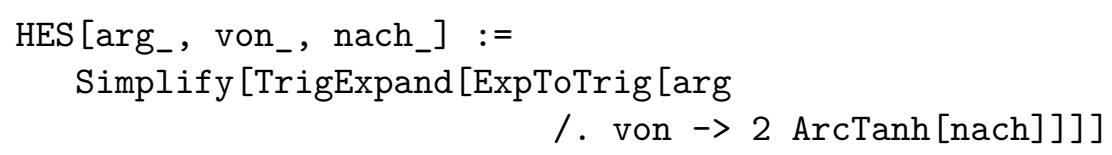

welche auch die Exponentialfunktionen bearbeiten kann. Im Verlaufe der verschiedenen Versionen hat sich die brauchbare Befehlsfolge mehrfach geändert.

\subsubsection{Maxima}

Die Funktion

HWS (arg, von, nach):= ratsimp (trigexpand (subst (2*atan (nach), von, arg)));

algebraisiert alle Winkelfunktionen. Die Befehle können natürlich auch interaktiv von innen nach außen ausgeführt werden. Ebenso funktioniert

HES (arg, von, nach):= ratsimp (trigexpand (subst ( $2 * \operatorname{atanh}$ (nach), von, arg))); 
für die hyperbolischen Funktionen, nicht aber die Exponentialfunktion. Der Ausdruck $e^{2 \text { atanh h }}$ lässt sich aber nur händisch vereinfachen. ${ }^{\square}$ Am einfachsten definiert man

$$
\operatorname{EXP}(x):=\cosh (x)+\sinh (x)
$$

und arbeitet von Anfang an mit dieser „neuen“ Funktion.

\subsubsection{Reduce}

Reduce hat die Simplifizierung der Ausdrücke eingebaut, das Verhalten wird durch voreingestellte Schalter (switches) gesteuert. Die Ausdrücke haben die Form

$$
\operatorname{sub}(a=2 * \operatorname{Atan}(t), \operatorname{Sin}(a)) ;
$$

und analog für die hyperbolischen Funktionen. Wieder funktioniert die Vereinfachung nicht für den Exponentialterm. Fordert man

for all $x$ let $\operatorname{Exp}(x)=\operatorname{Cosh}(x)+\operatorname{Sinh}(x)$;

so werden die entstehenden rationalen Ausdrücke auch mit

on factor;

passend gekürzt.

\subsubsection{Axiom}

Mit dem Befehl

$$
\text { normalize }(\exp (2 * \operatorname{atanh}(\mathrm{h})))
$$

und analog für die anderen Substitutionen führt man die Vereinfachung durch. Damit ist die Handhabung einfacher als in den anderen CAS. Leider ist Axiom nur unter Linux lauffähig.

\footnotetext{
${ }^{2}$ Zumindest habe ich es nicht hinbekommen. Für Hinweise bin ich dankbar.
} 


\section{Einleitung}

\subsection{Kleine Argumente}

Es kann mathematisch oder physikalisch sinnvoll sein, kleine Winkel oder Argumente der Exponentialfunktionen in der Grenze schon an den Symbolen identifizieren zu können.

Die in der Grenze der Identität entsprechenden Substitutionen

$\alpha=2 \arctan \left(\frac{t}{2}\right)=t+O\left(t^{3}\right) \quad$ und $\quad x=2 \operatorname{arctanh}\left(\frac{h}{2}\right)=h+O\left(h^{3}\right)$

sind gegenüber den ursprünglichen um den Faktor zwei modifiziert. Mit den Funktionen

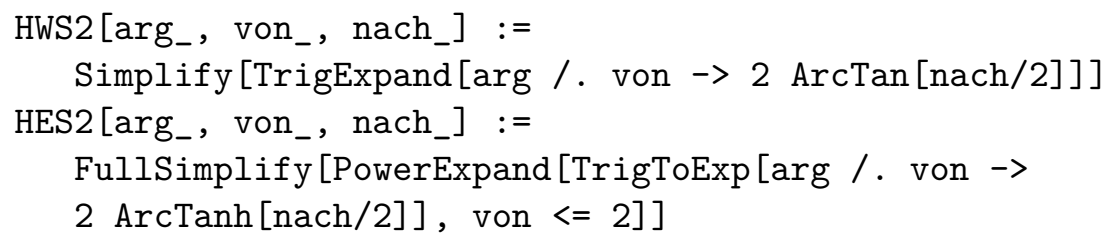

ergeben sich die folgenden Ersetzungen im Falle der Winkelfunktionen

$$
\begin{aligned}
\sin (\alpha) & =\frac{4 t}{4+t^{2}}=t-\frac{t^{3}}{4}+O\left(t^{5}\right) \\
\cos (\alpha) & =\frac{4-t^{2}}{4+t^{2}}=1-\frac{t^{2}}{2}+O\left(t^{4}\right) \\
\tan (\alpha) & =\frac{4 t}{4-t^{2}}=t+\frac{t^{3}}{4}+O\left(t^{5}\right) \\
\sin ^{2}(\alpha / 2) & =\frac{t^{2}}{4+t^{2}}=\frac{t^{2}}{4}+O\left(t^{4}\right) \\
\cos ^{2}(\alpha / 2) & =\frac{4}{4+t^{2}}=1-\frac{t^{2}}{4}+O\left(t^{4}\right)
\end{aligned}
$$


und im Falle der hyperbolischen Funktionen

$$
\begin{aligned}
\exp (x) & =\frac{2+h}{2-h}=1+h+\frac{h^{2}}{2}+O\left(h^{3}\right) \\
\exp (-x) & =\frac{2-h}{2+h}=1-h+\frac{h^{2}}{2}+O\left(h^{3}\right) \\
\sinh (x) & =\frac{4 h}{4-h^{2}}=h+\frac{h^{3}}{4}+O\left(h^{4}\right) \\
\cosh (x) & =\frac{4+h^{2}}{4-h^{2}}=1+\frac{h^{2}}{2}+O\left(h^{4}\right) \\
\tanh (x) & =\frac{4 h}{4+h^{2}}=h-\frac{h^{3}}{4}+O\left(h^{5}\right) .
\end{aligned}
$$

Diese Formeln haben den Vorteil, dass man bei den Umkehrsubstitutionen nicht mehr auf die Vorfaktoren aufpassen muss. Auch diese haben nun zwei Faktoren. Nebenbei haben wir mit Formel (‥33) gezeigt, dass

$$
x=2 \operatorname{arctanh}(h / 2)=\ln \left(\frac{2+h}{2-h}\right)
$$

An den generellen Aussagen dieser Arbeit ändert sich mit solchen Skalierungen nichts. 



\section{Anwendungen in der reinen Mathematik}

\subsection{Integration rationaler Funktionen}

Zur Integration einer rationalen Funktion $F(\alpha)=R(\sin \alpha, \cos \alpha)$ mit den Winkelfunktionen als Argumenten muss noch das Volumelement $d \alpha$ mittransformiert werden. Differentiation von $\alpha=2 \arctan t$ ergibt

$$
d \alpha=2 \frac{1}{1+t^{2}} d t
$$

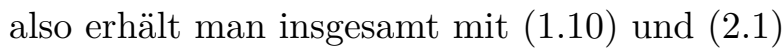

$$
\int R(\cos \alpha, \sin \alpha) d \alpha=\int R\left(\frac{1-t^{2}}{1+t^{2}}, \frac{2 t}{1+t^{2}}\right) \frac{2}{1+t^{2}} d t,
$$

wobei die Integration nun auf die einer rationalen Funktion zurückgeführt ist.

Der Nenner des Differentials wieder derselbe wie in den übrigen Sub-

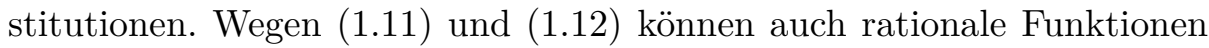
in den zusätzlichen Argumenten $\sin ^{2}(\alpha / 2)$ und $\cos ^{2}(\alpha / 2)$ rationalisiert werden.

Diese Substitution wird auf K. WeIERSTRAss zurückgeführt, und deshalb wird die Halbwinkelsubstitution im angelsächsischen Raum auch oft Weierstrass-Substitution genannt. Eine deutlich frühere Quelle findet sich aber bereits bei EuLER in "Institutiionum calculi integralis volumen primum, 1768, E342, Caput V, paragraph 261“, zu finden bei http://eulerarchive.maa.org/. Die erste Seite ist in Abb. 2. gezeigt.

Die Methode ist nicht unproblematisch, wie ein Paper von Jeffrey und RICH [.JR.94] zeigt: Schon beispielsweise das Integral

$$
\int \frac{3 d x}{5-4 \cos x}=\int \frac{6 d t}{1+9 t^{2}}=2 \arctan \left(3 \tan \frac{1}{2} x\right)
$$


2 Anwendungen in der reinen Mathematik

Problema 29 .

261. Formulae differentialis $\frac{\partial \Phi}{a+b \cos \Phi}$ integrale investigare.

Solutio.

Haec investigatio commodius institui nequit, quam ut formula propesita ad formam ordinariam reducatur, ponendo $\cos \phi=\frac{x-x x}{1+x x}$, ut rationaliter fiat $\sin \phi=\frac{2 x}{1+y x}$, hincque $\partial \phi \cos . \Phi=\frac{2 \partial x(1-x x)}{(1+x x)^{2}}$, sicque $\partial \phi=\frac{2 \partial x}{1+x x}$. Quia igitur $a+b \cos . \phi=\frac{a+b+(a-b) x x}{1+x x}$, erit formula nostra $\frac{\partial \phi}{a+b \cos \phi}=\frac{2 \partial x}{a+b+(a-b) x x}$, quae prout fuerit $a>b$ vel $a<b$, vel anguluin vel logarithmum praebet.

Casu $a>b$ reperitur casu $a<b$ vero est

$$
\int \frac{\partial \phi}{a+b \cos \phi}=\frac{a}{\sqrt{(a a-b b)}} \text { Arc. tang } \frac{(a-b) x}{\sqrt{(a a-b b)}}:
$$

Nunc vero est

$$
\int \frac{\partial \phi}{a+b \cos \cdot \phi}=\frac{x}{\sqrt{(b b-a} a)} l \frac{\gamma(b b-a c)+x(b-a)}{\gamma(b b-a a)-\frac{x(b-a)}{x(b-a)}} .
$$

$$
x=\sqrt{ } \frac{1-\cos .}{1+\cos .} \phi=\operatorname{tang} \cdot \frac{1}{2} \phi=\frac{\sin . \phi}{x+\cos . \phi} ;
$$

gua restitutione facta, cum sit

$$
\begin{aligned}
& 2 \text { Ang. tang. } \frac{(a-b)}{\gamma(a} \frac{x}{b b)}=\text { Ang. tang. } \frac{2 x v(a d-b b)}{a+b-(a-b) \times x} \\
& =\text { Ang. tang. } \frac{2 \sin \cdot \phi v(a a-b b)}{(a+b)(1+\cos . \phi)-(a-b)(1-\cos \cdot \bar{\phi})} \\
& =\text { Ang. tang. } \frac{\sin \cdot \phi \gamma(a a-b b)}{a \cos \phi+b} .
\end{aligned}
$$

- Quocirca pro casu $a>b$ adipiscimux:

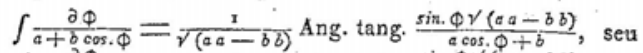

$$
\begin{aligned}
& \int \frac{\partial \Phi}{a+b \cos \phi}=\frac{1}{\sqrt{(a a-b \bar{b})}} \text { Ang. sin. } \frac{\sin \phi V(a a-b b)}{a+b \cos . \phi} \text {, sirc } \\
& \int \frac{\partial \phi}{a+b \cos . \Phi}=\frac{1}{\gamma(a a-b b)} \text { Ang. } \cos \frac{a \cos \cdot \phi+b}{a+b \cos . \phi} \text {. }
\end{aligned}
$$

Abbildung 2.1: Das erste Auftreten der Halbwinkelsubstitution bei EuLER.

zeigt Unstetigkeiten bei ungeraden Vielfachen von $\pi$, welche durch stufenförmige Wahl mehrerer Integrationskonstanten beseitigt werden können.

\subsection{Pythagoreische Zahlentripel}

Man betrachte noch einmal die Abb. 世.3. Die Länge $t$ auf der $y$-Achse sei rational, d.h.

$$
t=\frac{p}{q}
$$

mit $p, q$ teilerfremd und $p<q$. Dann folgt umgehend, dass der zugehörige Punkt $C$ auf dem Einheitskreis rationale Koordinaten $(x, y)$ hat, denn es 
gilt

$$
x=\sin \varphi=\frac{2 t}{1+t^{2}}=\frac{2 \frac{p}{q}}{1+\left(\frac{p}{q}\right)^{2}}=\frac{2 p q}{q^{2}+p^{2}}
$$

und

$$
y=\cos \varphi=\frac{1-t^{2}}{1+t^{2}}=\frac{1-\left(\frac{p}{q}\right)^{2}}{1+\left(\frac{p}{q}\right)^{2}}=\frac{q^{2}-p^{2}}{q^{2}+p^{2}} .
$$

Ferner gilt für die Zähler der eben gewonnenen Ausdrücke

$$
\begin{aligned}
\left(q^{2}-p^{2}\right)^{2}+(2 p q)^{2} & =q^{4}-2 q^{2} p^{2}+q^{4}+4 p^{2} q^{2}=q^{4}+2 q^{2} p^{2}+q^{4} \\
& =\left(q^{2}+p^{2}\right)^{2}
\end{aligned}
$$

und daher ist das Tripel

$$
\begin{aligned}
a & =2 p q \\
b & =q^{2}-p^{2} \\
c & =q^{2}+p^{2}
\end{aligned}
$$

immer eine ganzzahlige Lösung der Gleichung $a^{2}+b^{2}=c^{2}$. Umgekehrt gehört zu jedem solchen Pythagoreischen Zahlentripel nach Normieren auf die Hypothenuse $c$ ein rationaler Punkt $(x, y)$ mit $x=a / c$ und $y=b / c$ auf dem Einheitskreis.

Da es abzählbar unendlich viele Quotienten $t=p / q$ gibt, gibt es auch abzählbar unendlich viele rationale Punkte auf dem Einheitskreis sowie Pythagoreische Zahlentripel.

\subsection{Kosinussatz und Halbwinkelsatz}

Gegeben sei ein Dreieck in Einheitsdarstellung, vgl. Abb. ए2.

Ziel ist es, den Kosinussatz $c^{2}=a^{2}+b^{2}-2 a b \cos \gamma$ durch den Halbwinkel auszudrücken. Dazu löst man die Beziehung aus Gleichung (ㅁ. Kosinus nach dem Halbtangens auf. Mit den Abkürzungen $g:=\cos \gamma$ und $t:=\tan (\gamma / 2)$ formt man

$$
g=\frac{1-t^{2}}{1+t^{2}}
$$




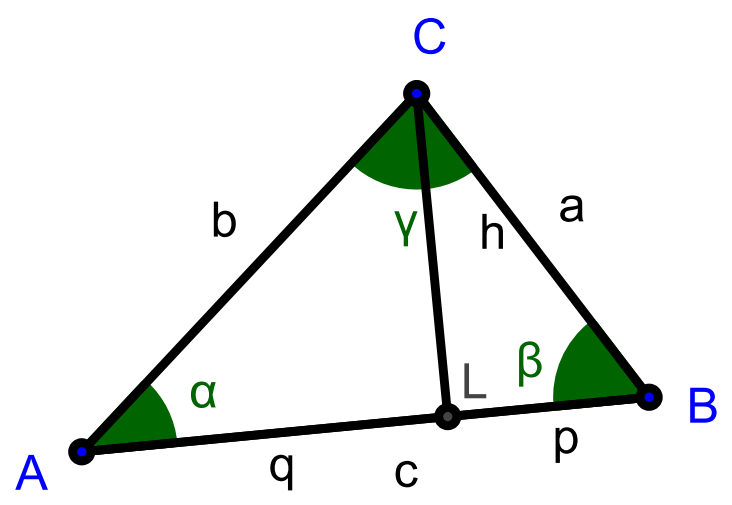

Abbildung 2.2: Das Einheitsdreieck mit den Standardbeschriftungen.

mittels $g\left(1+t^{2}\right)=1-t^{2}$ um zu $t^{2}(g+1)=1-g$ und löst dann nach $t^{2}$ auf

$$
t^{2}=\frac{1-g}{1+g}
$$

Setzt man nun darin nun den nach $g=\cos \gamma$ aufgelösten Kosinussatz

$$
g=\frac{a^{2}+b^{2}-c^{2}}{2 a b}
$$

ein, so erhält man mit

$$
\begin{aligned}
\tan ^{2}(\gamma / 2) & =\frac{1-\frac{a^{2}+b^{2}-c^{2}}{2 a b}}{1+\frac{a^{2}+b^{2}-c^{2}}{2 a b}}=\frac{2 a b-\left(a^{2}+b^{2}-c^{2}\right)}{2 a b+\left(a^{2}+b^{2}-c^{2}\right)} \\
& =\frac{c^{2}-(a-b)^{2}}{(a+b)^{2}-c^{2}}
\end{aligned}
$$

den gewünschten Kosinussatz in Halbwinkelformulierung. Dies ist der sog. Halbwinkelsatz: Mit $S:=(a+b+c) / 2$ lautet er in der Lehrbuchnotation

$$
\begin{aligned}
\tan ^{2}(\gamma / 2) & =\frac{(S-a)(S-b)}{S(S-c)} \\
& =\frac{(2 S-2 a)(2 S-2 b)}{2 S(2 S-c)}=\frac{(b+c-a)(a+c-b)}{(a+b+c)(a+b-c)}
\end{aligned}
$$


In beiden Notationen ist der Ausdruck auf der rechten Seite gleich.

\subsection{Höhere Vielfache}

Die Vielfachen der Winkelfunktionen sin und cos lassen sich wegen der Formel von Moivre (vgl. Abschnitt 2.4.D) durch eine Summe von Potenzen in genau diesen Winkelfunktionen darstellen. Daher wirkt die Halbwinkelsubstitution auch auf ganzzahlige Vielfache in den Argumenten der Winkelfunktionen. Unter Benutzung von Mathematica mit der Substitution

Simplify[TrigExpand $[\operatorname{Sin}[n \mathrm{x}] / . \mathrm{x} \rightarrow 2 \operatorname{ArcTan}[t]]]$

und analog für den Kosinus ergibt sich

$\left.\begin{array}{c|cc}n & \sin (n \alpha) & \cos (n \alpha) \\ \hline 1 & \frac{2 t}{1+t^{2}} & \frac{1-t^{2}}{1+t^{2}} \\ 2 & \frac{-4 t\left(-1+t^{2}\right)}{\left(1+t^{2}\right)^{2}} & \frac{1-6 t^{2}+t^{4}}{\left(1+t^{2}\right)^{2}} \\ 3 & \frac{6 t-20 t^{3}+6 t^{5}}{\left(1+t^{2}\right)^{3}} & \frac{1-15 t^{2}+15 t^{4}-t^{6}}{\left(1+t^{2}\right)^{3}} \\ 4 & \frac{-8 t\left(-1+7 t^{2}-7 t^{4}+t^{6}\right)}{\left(1+t^{2}\right)^{4}} & \frac{1-28 t^{2}+70 t^{4}-28 t^{6}+t^{8}}{\left(1+t^{2}\right)^{4}} \\ 5 & \frac{2 t\left(5-60 t^{2}+126 t^{4}-60 t^{6}+5 t^{8}\right)}{\left(1+t^{2}\right)^{5}} & \frac{1-45 t^{2}+210 t^{4}-210 t^{6}+45 t^{8}-t^{10}}{\left(1+t^{2}\right)^{5}}\end{array}\right\}$

Ferner zeigen die Formeln durch den Übergang $n \alpha=: \varphi$, dass mit den Substitutionen $t_{4}:=\tan \left(\frac{\varphi}{4}\right), t_{6}:=\tan \left(\frac{\varphi}{6}\right)$ usw. analoge Rationalisierungen der Winkelfunktionen in $\varphi$ möglich sind.

Mit den komplexen Methoden des folgenden Abschnittes kann man geschlossene Ausdrücke für die Koeffizienten angeben:

$$
\begin{aligned}
& \sin (n \alpha)=\frac{1}{\left(1+t^{2}\right)^{n}} \sum_{j=0}^{n-1}\left(\begin{array}{c}
2 n \\
2 j+1
\end{array}\right)(-1)^{j} t^{2 j+1} \\
& \cos (n \alpha)=\frac{1}{\left(1+t^{2}\right)^{n}} \sum_{j=0}^{n}\left(\begin{array}{c}
2 n \\
2 j
\end{array}\right)(-1)^{j} t^{2 j} .
\end{aligned}
$$


2 Anwendungen in der reinen Mathematik

\subsubsection{Die Koeffizienten der höheren Vielfachen}

Im Komplexen gilt für die höheren Vielfache die Formel von MoIvRE

$$
\cos (n \alpha)+i \sin (n \alpha)=\exp (i n \alpha)=\exp (i \alpha)^{n}=(\cos (\alpha)+i \sin (\alpha))^{n},
$$

welche vermöge der Halbwinkelsubstitution (ㅁ.

$$
\begin{aligned}
\cos (n \alpha)+i \sin (n \alpha) & =\left(\frac{1-t^{2}}{1+t^{2}}+i \frac{2 t}{1+t^{2}}\right)^{n} \\
& =\frac{1}{\left(1+t^{2}\right)^{n}}\left(1-t^{2}+i 2 t\right)^{n} \\
& =\frac{1}{\left(1+t^{2}\right)^{n}}(1+i t)^{2 n} .
\end{aligned}
$$

Mit dem Binomischen Satz erhält man weiter

$$
\begin{aligned}
\cos (n \alpha)+i \sin (n \alpha) & =\frac{1}{\left(1+t^{2}\right)^{n}} \sum_{k=0}^{\infty}\left(\begin{array}{c}
2 n \\
k
\end{array}\right)(i t)^{k} \\
& =\frac{1}{\left(1+t^{2}\right)^{n}} \sum_{k=0}^{2 n}\left(\begin{array}{c}
2 n \\
k
\end{array}\right)(i t)^{k},
\end{aligned}
$$

die Summe bricht also ab. Nun spaltet man die Summanden in gerade und ungerade Indizes auf und erhält

$$
\begin{aligned}
& \cos (n \alpha)+i \sin (n \alpha) \\
& =\frac{1}{\left(1+t^{2}\right)^{n}}\left[\sum_{j=0}^{n}\left(\begin{array}{c}
2 n \\
2 j
\end{array}\right)(i t)^{2 j}+\sum_{j=0}^{n-1}\left(\begin{array}{c}
2 n \\
2 j+1
\end{array}\right)(i t)^{2 j+1}\right] \\
& =\frac{1}{\left(1+t^{2}\right)^{n}}\left[\sum_{j=0}^{n}\left(\begin{array}{c}
2 n \\
2 j
\end{array}\right) i^{2 j} t^{2 j}+\sum_{j=0}^{n-1}\left(\begin{array}{c}
2 n \\
2 j+1
\end{array}\right) i^{2 j+1} t^{2 j+1}\right] \\
& =\frac{1}{\left(1+t^{2}\right)^{n}}\left[\sum_{j=0}^{n}\left(\begin{array}{c}
2 n \\
2 j
\end{array}\right)(-1)^{j} t^{2 j}+\sum_{j=0}^{n-1}\left(\begin{array}{c}
2 n \\
2 j+1
\end{array}\right) i(-1)^{j} t^{2 j+1}\right] .
\end{aligned}
$$

Vergleich von Real- und Imaginärteil liefert nun die Formeln ([2.]) und (ए..2).

Ohne den Durchgang durchs Komplexe beweist man ebenso, dass

$$
\cosh (n x)+\sinh (n x)=\exp (n x)=\exp (x)^{n}=(\cosh x+\sinh x)^{n},
$$


was vermöge der neuen Substitutionen ([.22) und ([.2.3) übergeht in

$$
\begin{aligned}
\cosh (n x)+\sinh (n x) & =\left(\frac{1+h^{2}}{1-h^{2}}+\frac{2 h}{1-h^{2}}\right)^{n} \\
& =\frac{1}{\left(1-h^{2}\right)^{n}}(1+h)^{2 n} .
\end{aligned}
$$

Wieder mit dem Binomischen Satz erhält man die endliche Summe

$$
\cosh (n x)+\sinh (n x)=\frac{1}{\left(1-h^{2}\right)^{n}} \sum_{k=0}^{2 n}\left(\begin{array}{c}
2 n \\
k
\end{array}\right) h^{k} .
$$

Nun teilt man die linken und rechten Seiten in gerade und ungerade Funktionen von $h$ auf und erhält schließlich

$$
\begin{aligned}
\cosh (n 2 \operatorname{arctanh}(h)) & =\frac{1}{\left(1-h^{2}\right)^{n}} \sum_{k=0}^{n}\left(\begin{array}{l}
2 n \\
2 k
\end{array}\right) h^{2 k} \\
\sinh (n 2 \operatorname{arctanh}(h)) & =\frac{1}{\left(1-h^{2}\right)^{n}}+\sum_{j=0}^{n-1}\left(\begin{array}{c}
2 n \\
2 k+1
\end{array}\right) h^{2 k+1} .
\end{aligned}
$$

Mit den vierteln, achteln usw. Argumenten rationalisiert man auch hier wieder die Funktionen.

\subsection{Die Einheitswurzeln}

In diesem Abschnitt soll eine Antwort versucht werden auf die Frage, warum bestimmte Werte von Winkelfunktionen besonders „einfach“ sind. Beispielsweise ist

$$
\sin \left(\frac{7 \pi}{12}\right)=\frac{1+\sqrt{3}}{2 \sqrt{2}}
$$

Zur Erinnerung sei erwähnt, dass man mit dem Höhensatz die Wurzel einer beliebigen Zahl $x$ ziehen kann. In den Standardbezeichnungen, vgl. wieder Abb. [2.2, gilt $h^{2}=p q$, man wählt also $p=x$ und $q=1$. Indem man analog eine der beteiligten Strecken an den Strahlensätzen geschickt zu 1 wählt, gelingt die Multiplikation oder Division beliebiger Zahlen mit geometrischen Methoden. Damit ist eine Zahl mit Zirkel und Lineal konstruierbar, wenn sie aus ganzen Zahlen und deren Wurzeln sowie Produkten und Quotienten davon besteht. 
Die $n$-ten Einheitswurzeln sind die komplexen Lösungen von $z^{n}=1$, also mit $\zeta_{n}:=\exp \left(\frac{2 \pi i}{n}\right)$ die Zahlen $\zeta_{n}, \zeta_{n}^{2}, \zeta_{n}^{3}, \ldots \zeta_{n}^{n}=1$. Geometrisch bilden diese ein regelmäßiges $n$-Eck mit dem Einheitskreis als Umkreis und einer Ecke auf der reellen 1.

Gegeben sei ein regelmäßiges n-Eck auf dem Einheitskreis mit dem Punkt $(1,0)$ als einer Ecke, vgl. Abb. [2.3.

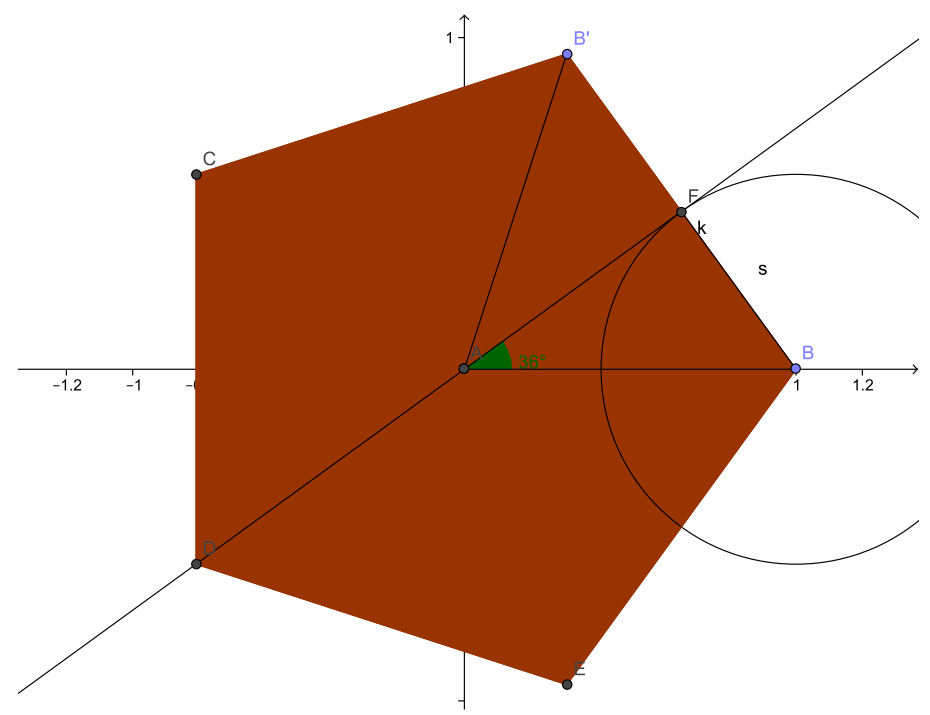

Abbildung 2.3: Die fünften Einheitswurzeln. Sei $\alpha=\frac{360^{\circ}}{n}$ der Innenwinkel des regelmäßigen n-Ecks. Hat die Strecke $s=\sin \frac{\alpha}{2}$ eine mit Zirkel und Lineal konstruierbare Länge, so ist genau dann das regelmäßige n-Eck mit Zirkel und Lineal konstruierbar. Denn: Schlage einen Kreis $k$ mit Radius $s$ um $B$ und konstruiere die Tangente von $A$ an $k$. Die Strecke $s$ steht senkrecht auf dieser Tangente. Verdoppele $s$ und wiederhole die gesamte Konstruktion um den Punkt $B^{\prime}$.

Dann sind alle Ecken gegeben durch die Winkel $\alpha=2 \pi \frac{m}{n}$ und die Koordinaten $\left(\cos 2 \pi \frac{m}{n}, \sin 2 \pi \frac{m}{n}\right)$ mit $m \leq n$ ganze Zahlen. Insbesondere folgt

$$
\sin \left(n \frac{\alpha}{2}\right)=\sin \pi m=0
$$

Gesucht sind damit die Nullstellen einer Tabelle analog zum Kapitel ए.4. Wir erhalten die ersten Glieder zu 


\subsection{Die Einheitswurzeln}

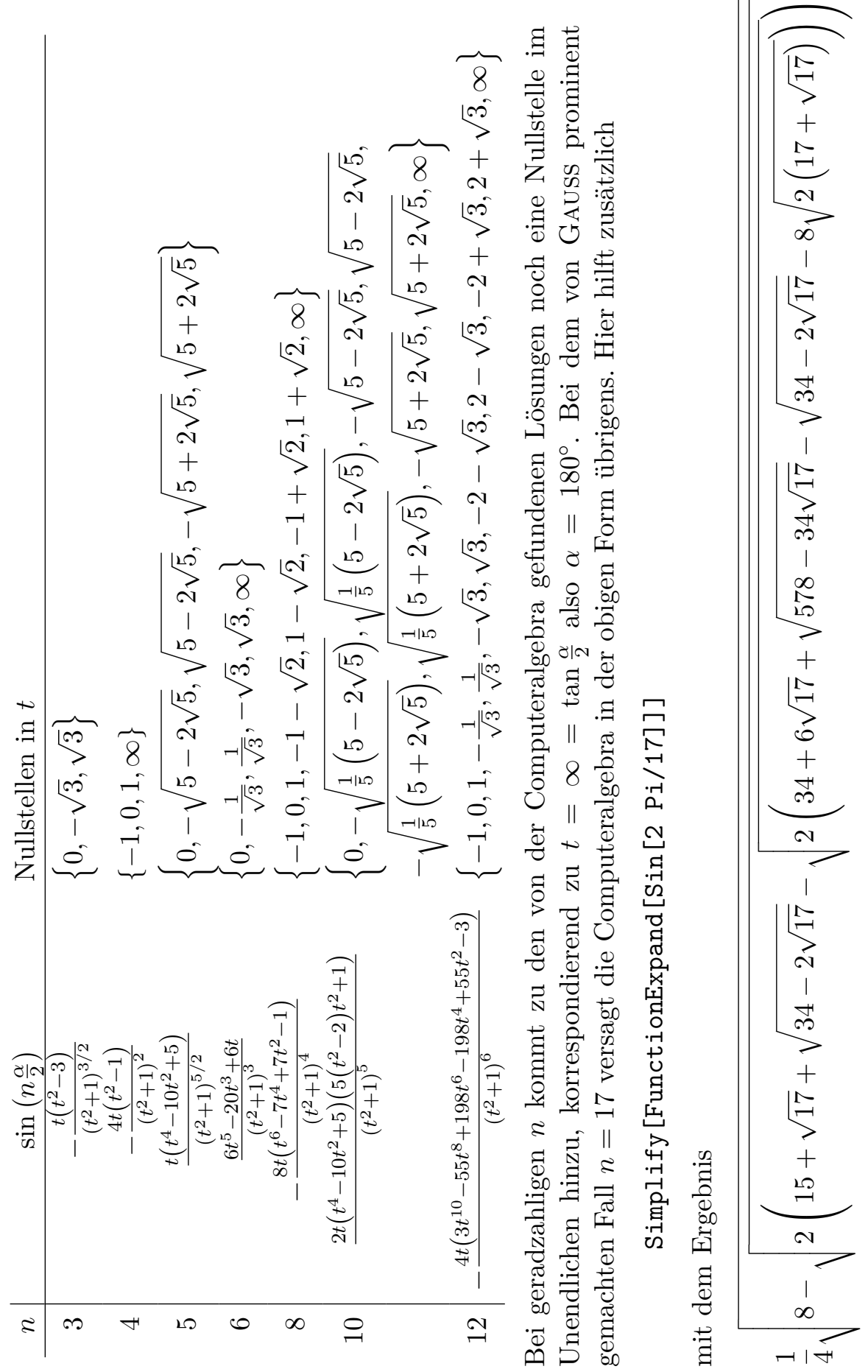


Daher sind die Winkelfunktionen der Einheitswurzeln Nullstellen von Polynomen mit ganzzahligen Koeffizienten. Solche Zahlen nennt man algebraisch. Die bekannten mit Zirkel und Lineal konstruierbaren $n$-Ecke kleiner als 20 sind: 3, 4, 5, 6, 8, 10, 12, 15, 16, 17, und 20. Genauer dürfen in der Primzahlzerlegung von $n$ nur die Zahlen 2, 3, 5, 17, 257 und 65537 vorkommen, außer der 2 alles Fermatsche Primzahlen.

\subsection{Eine stereografische Projektion}

Die Abbildung ‥3 stellt eine zweidimensionale stereografische Projektion dar, welche den reellen Zahlenstrahl auf den Einheitskreis abbildet. Dazu dreht man sie um $90^{\circ}$ und erhält die Abb. ‥4.

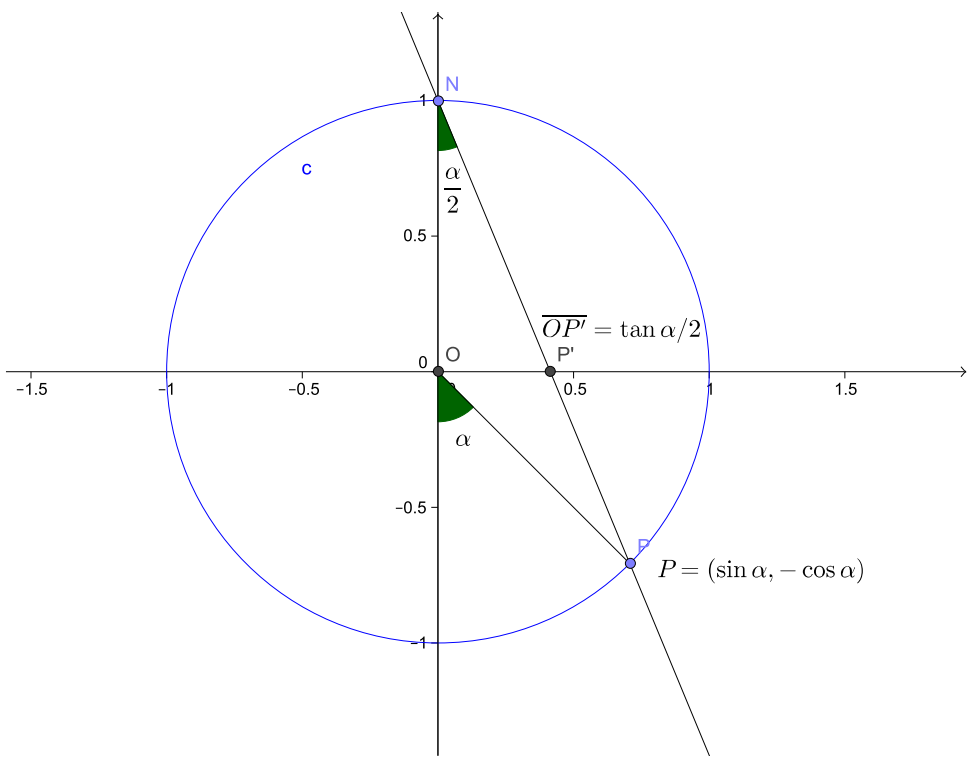

Abbildung 2.4: Die Halbwinkelsubstitution als stereografische Projektion.

Ein Punkt $P$ des Einheitskreises um den Ursprung wird vom Nordpol $N$ des Kreises entlang der Gerade $N P$ auf den Punkt $P^{\prime}$ der $x$-Achse abgebildet. Die Länge der Strecke $\overline{O P^{\prime}}$ ist dann gleich $\tan \frac{\alpha}{2}$. Der Nordpol selbst entspricht dann dem unendlich fernen Punkt. Daher ist die Kenntnis der Koordinaten des Einheitskreises, also die von $\sin \alpha$ und $\cos \alpha$, gleichwertig mit der Kenntnis von $t=\tan \frac{\alpha}{2}$. 


\subsubsection{In drei Dimensionen}

Die eigentliche stereografische Projektion bildet die komplexe Zahlenebene auf die Einheitskugel ab. Die Projektionsebene steht dabei senkrecht auf der Nord-Süd-Achse der Kugel. Gegeben sei die Kugel $\boldsymbol{k}=(x, y, z)^{T}$ um den Ursprung mit der Gleichung $x^{2}+y^{2}+z^{2}=R^{2}$. Der Nordpol der Kugel $\boldsymbol{n}=(0,0, R)^{T}$ sowie die aufeinander abzubildenden Punkte $\boldsymbol{k}$ der Kugel und $\boldsymbol{p}=(\xi, \eta, a)^{T}$ der Ebene liegen dabei auf einer Geraden. Die Abbildungsgleichungen erhält man als Lösung von $\boldsymbol{p}=\boldsymbol{n}-\lambda(\boldsymbol{n}-\boldsymbol{k})$ nach $(\xi, \eta, \lambda)$. Sie lauten nach Einsetzen von $\lambda$

$$
\xi=\frac{(R-a) x}{R-z} \quad \text { und } \quad \eta=\frac{(R-a) y}{R-z} .
$$

Die inversen Gleichungen lauten unter Benutzung der Kugelgleichung

$$
\begin{aligned}
x & =\frac{2 \xi R(R-a)}{(R-a)^{2}+\eta^{2}+\xi^{2}} \\
y & =\frac{2 \eta R(R-a)}{(R-a)^{2}+\eta^{2}+\xi^{2}} \\
z & =\frac{R\left(-(R-a)^{2}+\eta^{2}+\xi^{2}\right)}{(R-a)^{2}+\eta^{2}+\xi^{2}} .
\end{aligned}
$$

Verläuft die Projektionsebene durch den Kugelmittelpunkt, so ist $a=0$. Ist der Südpol Element der Ebene, so ist $a=-R$.

\subsection{Inversion von Polarkoordinaten}

Gegeben seien nun ebene Polarkoordinaten, also $x(r, \alpha)=r \cos \alpha$ und $y(r, \alpha)=r \sin \alpha$. Gesucht sind nun die Umkehrtransformationen $r(x, y)$ und $\alpha(x, y)$.

In nahezu sämtlichen Formelsammlungen findet man dazu die Formeln

$$
r=\sqrt{x^{2}+y^{2}} \quad \text { und } \quad \alpha=\arctan \frac{y}{x} .
$$

Diese Formel liefert aber nur im Bereich $\alpha \in(-\pi / 2, \pi / 2)$ Werte, so dass mit $r>0$ nur die rechte Halbebene erfasst wird.

Dieses Problem verschwindet, wenn man den halben Winkel verwendet. Die Formeln (‥9) liefern sofort mit $r=\sqrt{x^{2}+y^{2}}$ erweitert

$$
t=\tan \left(\frac{\alpha}{2}\right)=\frac{\sin \alpha}{1+\cos \alpha}=\frac{r \sin \alpha}{r+r \cos \alpha}=\frac{y}{\sqrt{x^{2}+y^{2}}+x}
$$


2 Anwendungen in der reinen Mathematik

oder

$$
t=\tan \left(\frac{\alpha}{2}\right)=\frac{1-\cos \alpha}{\sin \alpha}=\frac{r-r \cos \alpha}{r \sin \alpha}=\frac{\sqrt{x^{2}+y^{2}}-x}{y} .
$$

Die letzte Formel ist undefiniert für $y=0$, also auf der $x$-Achse. Daher wird die erste verwendet, welche nur auf der negativen $x$-Achse Schwierigkeiten macht, entsprechend $\alpha= \pm \pi$. Diese Formel (ए.3D) ist in diversen Programmiersprachen als ATAN2 als Funktion zweier Argumente implementiert. In Abb. 2.5 ist eine solche Implementation in Mathematica gezeigt.
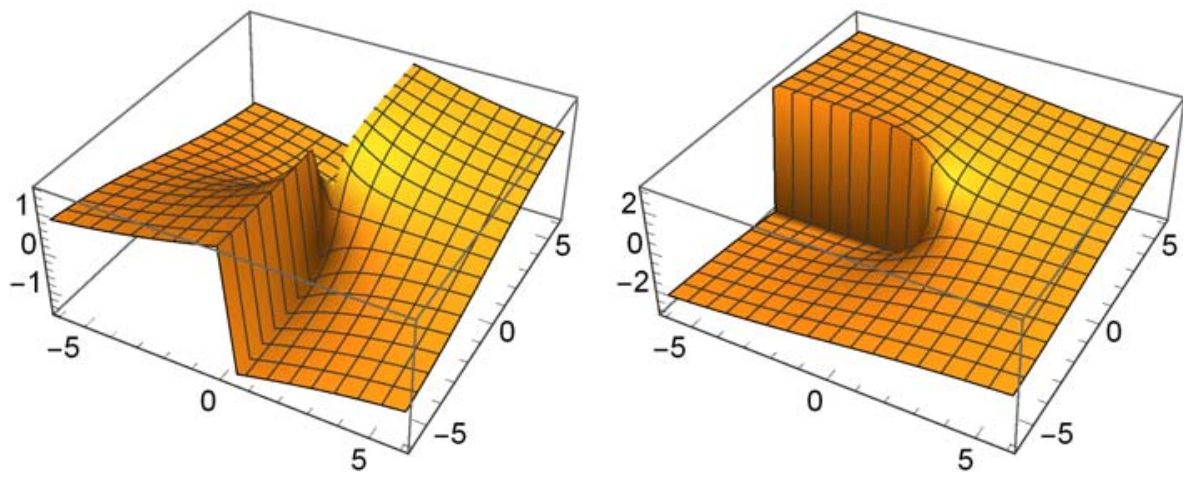

Abbildung 2.5: Der Arcustangens von einem $\arctan (y / x)($ (ए.30) und zwei Argumenten $\arctan (x, y)($ (2.3D) in Mathematica. In manchen Programmiersprachen ist die Reihenfolge der Argumente vertauscht.

\subsection{Inversion von Kugelkoordinaten}

Gegeben seien Kugelkoordinaten, also

$$
\begin{aligned}
& x=r \cos (\alpha) \sin (\theta) \\
& y=r \sin (\alpha) \sin (\theta) \\
& z=r \cos (\theta) .
\end{aligned}
$$

Die Substitution $a=\tan \left(\frac{\alpha}{2}\right)$ und $t=\tan \left(\frac{\theta}{2}\right)$. 


$$
\begin{aligned}
& \mathrm{xr}=\text { Simplify }[\operatorname{Tr} i g E x p a n d[\mathrm{x} / .\{\backslash[\mathrm{Alpha}] \rightarrow 2 \operatorname{ArcTan}[\mathrm{a}], \\
& \backslash[\text { Theta] } \rightarrow \text { 2ArcTan }[\mathrm{t}]\}]] \\
& \text { yr }=\text { Simplify }[\operatorname{TrigExpand}[\mathrm{y} / .\{\backslash[\mathrm{Alpha}] \rightarrow 2 \operatorname{ArcTan}[\mathrm{a}], \\
& \backslash \text { [Theta] } \rightarrow 2 \operatorname{ArcTan}[t]\}]] \\
& \mathrm{zr}=\text { Simplify }[\operatorname{TrigExpand}[z / .\{\backslash[\mathrm{Alpha}] \rightarrow 2 \operatorname{ArcTan}[\mathrm{a}], \\
& \backslash[\text { Theta }] \rightarrow \text { 2ArcTan }[\mathrm{t}]\}]]
\end{aligned}
$$

führt auf die rationalen Funktionen

$$
\begin{aligned}
& x_{r}=\frac{-2\left(-1+a^{2}\right) r t}{\left(1+a^{2}\right)\left(1+t^{2}\right)} \\
& y_{r}=\frac{4 a r t}{\left(1+a^{2}\right)\left(1+t^{2}\right)} \\
& z_{r}=\frac{r-r t^{2}}{1+t^{2}} .
\end{aligned}
$$

Das Gleichungssystem

$$
\begin{aligned}
& \text { Simplify [Solve }[\{\mathrm{xr}==\mathrm{X}, \mathrm{yr}==\mathrm{Y}, \mathrm{zr}==\mathrm{Z} \text {, } \\
& \left.\left.\left.\mathrm{r}^{\wedge} 2==\mathrm{X}^{\wedge} 2+\mathrm{Y}^{\wedge} 2+\mathrm{Z}^{\wedge} 2\right\},\{\mathrm{a}, \mathrm{t}\}\right]\right]
\end{aligned}
$$

hat schließlich die beiden Lösungen

$$
\begin{aligned}
a & =\frac{-\left(X \sqrt{X^{2}+Y^{2}}\right) \pm(r+Z) \sqrt{2 r^{2}-X^{2}-Y^{2}-2 r Z}}{Y \sqrt{X^{2}+Y^{2}}} \\
t & = \pm \frac{\sqrt{2 r^{2}-X^{2}-Y^{2}-2 r Z}}{\sqrt{X^{2}+Y^{2}}}
\end{aligned}
$$

\subsection{Eine Verallgemeinerung auf zwei Argumente}

Das Additionstheorem des Tangens zweier Argumente kann aus denen für den Sinus (ㅁ. $)$ und den Kosinus (ㅍ. 2 ) hergeleitet werden. Es lautet

$$
\tan (\alpha \pm \beta)=\frac{\tan \alpha \pm \tan \beta}{1 \mp \tan \alpha \tan \beta}
$$

also mit den Abkürzungen $t:=\tan \alpha / 2$ und $u:=\tan \beta / 2$ für den halben Winkel

$$
\tan \left(\frac{\alpha \pm \beta}{2}\right)=\frac{t \pm u}{1 \mp t u}
$$




\section{Anwendungen in der reinen Mathematik}

Damit soll nun gezeigt werden, dass gilt

$$
\tan \left(\frac{\alpha \pm \beta}{2}\right)=\frac{\sin \alpha+\sin \beta}{\cos \alpha+\cos \beta}=-\frac{\cos \alpha-\cos \beta}{\sin \alpha-\sin \beta} .
$$

Die beiden rechten Seiten verifiziert man durch Einsetzen der Halbwinkelsubstitution. Für den ersten Term gilt

$$
\frac{\sin \alpha+\sin \beta}{\cos \alpha+\cos \beta}=\frac{\frac{2 t}{1+t^{2}}+\frac{2 u}{1+u^{2}}}{\frac{1-t^{2}}{1+t^{2}}+\frac{1-u^{2}}{1+u^{2}}}=\frac{2 t\left(1+u^{2}\right)+2 u\left(1+t^{2}\right)}{\left(1-t^{2}\right)\left(1+u^{2}\right)+\left(1-u^{2}\right)\left(1+t^{2}\right)} .
$$

Nach Ausmultiplizieren heben sich einige Terme weg, und es bleibt übrig

$$
\%=\frac{2\left(t+u+t u^{2}+u t^{2}\right)}{2\left(1-u^{2} t^{2}\right)}=\frac{u(1+u t)+t(1+u t)}{(1-u t)(1+u t)}=\frac{u+t}{1-u t},
$$

letzteres nach Kürzen von $(1+u t)$. Für den zweiten Term gilt

$$
-\frac{\cos \alpha-\cos \beta}{\sin \alpha-\sin \beta}=-\frac{\frac{1-t^{2}}{1+t^{2}}-\frac{1-u^{2}}{1+u^{2}}}{\frac{2 t}{1+t^{2}}-\frac{2 u}{1+u^{2}}}=-\frac{\left(1-t^{2}\right)\left(1+u^{2}\right)-\left(1-u^{2}\right)\left(1+t^{2}\right)}{2 t\left(1+u^{2}\right)-2 u\left(1+t^{2}\right)} .
$$

Wieder heben sich nach Ausmultiplizieren einige Terme weg, und man erhält

$$
\%=-\frac{2\left(u^{2}-t^{2}\right)}{2\left(t+t u^{2}-u-u t^{2}\right)}=-\frac{(u-t)(u+t)}{(t-u)(1-u t)}=\frac{u+t}{1-u t},
$$

diesmal nach Kürzen von $(u-t)$.

Damit sind die Formeln (2.37) bewiesen. Man erhält aus ihnen als Spezialfälle für $\alpha=0$ oder $\beta=0$ die Formeln (ㅁ. $)$ zurück.

\subsection{Die erste Eisenstein-Funktion}

Nun soll eine weitere Methode gezeigt werden, mit der die Trigonometrischen Funktionen aus dem Halbtangens hergeleitet werden können. Ich folge hier teilweise [Rem.92], Kap $11 \S 4^{*}$.

Betrachtet man den Kehrwert des Sinus, so hat auch dieser mit (‥JU) eine rationale Gestalt, und zwar

$$
\frac{1}{\sin z}=\frac{1}{2}\left(\frac{1+t^{2}}{t}\right)=\frac{1}{2}\left(\frac{1}{t}+t\right)=\frac{1}{2}\left(\cot \frac{z}{2}+\tan \frac{z}{2}\right) .
$$


Durch Hinzunahme des Kehrwertes kann man die quadratischen Terme vermeiden. Ferner kann man die Argumente translatieren, und erhält

$$
\frac{1}{\sin z}=\frac{1}{2}\left(\cot \frac{z}{2}-\cot \frac{z+\pi}{2}\right)
$$

Damit ist der Cosecans, also der Kehrwert des Sinus, auf eine Summe mit Summanden aus einer einzigen periodische Funktion mit geeignet verschobenen Argumenten zurückgeführt. Die erste EisEnstEIn-Funktion ist nun genau

$$
\epsilon_{1}(z):=\pi \cot (\pi z)
$$

und man kann

$$
\frac{\pi}{\sin (\pi z)}=\frac{1}{2}\left[\epsilon_{1}\left(\frac{z}{2}\right)-\epsilon_{1}\left(\frac{z+1}{2}\right)\right]
$$

als Definition des Sinus auffassen. Wegen $\cos (\pi z)=\sin (\pi(z+1 / 2))$ hat man dann ebenso

$$
\frac{\pi}{\cos (\pi z)}=\frac{1}{2}\left[\epsilon_{1}\left(\frac{2 z+1}{4}\right)-\epsilon_{1}\left(\frac{2 z+3}{4}\right)\right] .
$$

Natürlich kann man wieder die Exponentialfunktion im Komplexen durch den Halbtangens ausdrücken. Für $n=1$ erhält man aus (ए.J6) die Gleichung

$$
\begin{aligned}
\exp (i \alpha) & =\cos (\alpha)+i \sin (\alpha)=\frac{(1+i t)^{2}}{1+t^{2}}=\frac{(1+i t)^{2}}{(1+i t)(1-i t)}=\frac{1+i t}{1-i t} \\
& =\frac{i-t}{i+t}
\end{aligned}
$$

Mit der Substitution $\alpha=2 \pi z$ erhält man wegen $t(\alpha)=\pi / \epsilon_{1}(z)$ in den Termen der ersten Eisenstein-Funktion

$$
\exp (2 \pi i z)=\exp (i \alpha)=\frac{1+i t}{1-i t}=\frac{\epsilon_{1}(z)+\pi i}{\epsilon_{1}(z)-\pi i} .
$$

Der tiefere Grund für das Funktionieren der Halbwinkelsubstitution liegt also tatsächlich in der Funktionentheorie begründet. 


\subsection{Eine Differentialgleichung vom Riccati-Typ}

Die Ableitung von $t=\tan (\alpha / 2)$ nach dem Argument $\alpha$ ist, mit den Abkürzungen $s_{2}:=\sin (\alpha / 2)$ und $c_{2}:=\cos (\alpha / 2)$

$$
t^{\prime}=\left(\frac{s_{2}}{c_{2}}\right)^{\prime}=\frac{s_{2}^{\prime} c_{2}-s_{2} c_{2}^{\prime}}{c_{2}^{2}}=\frac{1}{2} \frac{c_{2}^{2}+s_{2}^{2}}{c_{2}^{2}}=\frac{1}{2}\left(1+t^{2}\right)
$$

Diese Gleichung kann als Differentialgleichung

$$
t^{\prime}=\frac{1}{2}\left(1+t^{2}\right)
$$

verstanden werden. Durch Trennung der Veränderlichen erhält man

$$
2 \int \frac{d t}{1+t^{2}}=\int d \alpha
$$

also $2 \arctan t=\alpha-\alpha_{0}$ und damit $t=\tan \left(\frac{\alpha-\alpha_{0}}{2}\right)$. Wählt man $\alpha_{0}=$ $\alpha_{1}+\pi$, so ist auch $t=-\cot \left(\frac{\alpha-\alpha_{1}}{2}\right)$ Lösung der DGL. Im Komplexen kommen noch die trivialen Lösungen $t= \pm i$ hinzu, welche aber wegen $\arctan ( \pm i)= \pm i \infty$ im Reellen keine Bedeutung besitzen.

Die betrachtete DGL ist gleichzeitig ein Sonderfall der (im Allgemeinen nicht geschlossen lösbaren) RICCATIschen DGL

$$
t^{\prime}+g(\alpha) t+h(\alpha) t^{2}=k(\alpha)
$$

so dass das Auftauchen solcher Typen von DGLn bei Problemen vermutet werden darf, welche mit der Halbwinkelsubstitution vereinfacht beschrieben werden können.

Die Transformation

$$
u=\exp \int h(\alpha) t(\alpha) d \alpha
$$

überführt diese Gleichung in eine lineare Differentialgleichung zweiter Ordnung, denn es gilt (vgl. [Wal.90])

$$
u^{\prime \prime}+u^{\prime}\left(g-\frac{h^{\prime}}{h}\right)-u k h=u h\left(h t^{2}+t^{\prime}+g t-k\right)=0 \quad .
$$

Diese kann ihrerseits wieder durch Einführung von $v=u^{\prime}$ als System zweier linearer Differentialgleichungen erster Ordnung in $u$ und $v$ geschrieben 
werden. In unserem Beispiel gilt $h=-1 / 2, k=1 / 2$ und $g=0$, womit $u=\cos (\alpha / 2)$ der DGL $u^{\prime \prime}+u / 4=0$ genügt.

Wir beschreiben eine Drehung mit der linearen Differentialgleichung

$$
\left(\begin{array}{l}
x^{\prime}(t) \\
y^{\prime}(t) \\
z^{\prime}(t)
\end{array}\right)=\left(\begin{array}{l}
p(t) \\
q(t) \\
r(t)
\end{array}\right) \times\left(\begin{array}{l}
x(t) \\
y(t) \\
z(t)
\end{array}\right)
$$

welche in Komponenten lautet

$$
\begin{array}{rlrl}
x^{\prime} & = & & r y-q z \\
y^{\prime} & = & -r x & +p z \\
z^{\prime} & = & q x-p y
\end{array}
$$

Zur Lösung dieses Systems von DGLn schaut man natürlich in den KAMKE [Kam77] und wird unter der Nummer 8.50 fündig. Aus der Antisymmetrie des Kreuzproduktes folgt

$$
x x^{\prime}+y y^{\prime}+z z^{\prime}=0,
$$

also ist

$$
x^{2}+y^{2}+z^{2}=\text { const } .
$$

Wegen der Linearität der Differentialgleichung gehen die Lösungen zu verschiedenen Konstanten aus der von

$$
x^{2}+y^{2}+z^{2}=1
$$

durch Skalierung hervor. Wir beschränken uns daher im Folgenden auf diesen Fall.

Nun benutzen wir die komplexen Substitutionen von DARBoux [Dar14] (S. 27-31)

$$
\begin{aligned}
\xi & =\frac{x+i y}{1-z}=\frac{1+z}{x-i y} \\
-\frac{1}{\eta} & =\frac{x-i y}{1-z}=\frac{1+z}{x+i y}
\end{aligned}
$$

mit den Umkehrungen

$$
\begin{aligned}
& x=\frac{1-\xi \eta}{\xi-\eta} \\
& y=i \frac{1+\xi \eta}{\xi-\eta} \\
& z=\frac{\xi+\eta}{\xi-\eta} .
\end{aligned}
$$


Die Verwandtschaft mit der räumlichen stereografischen Projektion ([.26) ist unverkennbar. Diese Umkehrungen und ihre Ableitungen setzen wir nun in die Gleichungen (2.46]) bis (2.48) ein. Anschließend bringen wir diese Ausdrücke auf die Form $(*)=0$. Die so erhaltenen Gleichungen sollen mit $(x),(y)$ und $(z)$ bezeichnet werden. Die Linearkombination $(z)-\xi((x)+i(y))$ führt nach Kürzen von $\xi-\eta$ auf die RiccAtische Differentialgleichung

$$
\xi^{\prime}=\frac{q-i p}{2}-i r \xi+\frac{q+i p}{2} \xi^{2},
$$

und die Linearkombination $(z)-\eta((x)+i(y))$ führt wieder nach Kürzen von $\xi-\eta$ auf dieselbe Diffentialgleichung in $\eta$.

In Mathematica gelingt beides problemlos mit

\section{FullSimplify [Reduce [DGK, $\{\backslash[\mathrm{Xi}],[\mathrm{t}], \backslash[\mathrm{Eta}],[\mathrm{t}]\}]]$,}

wobei DGK das substituierte System der Differentialgleichungen ist. Diese Reduktion kann auch auf die inhomogene Verallgemeinerung

$$
\left(\begin{array}{l}
x^{\prime}(t) \\
y^{\prime}(t) \\
z^{\prime}(t)
\end{array}\right)=\left(\begin{array}{c}
p(t) \\
q(t) \\
r(t)
\end{array}\right) \times\left(\begin{array}{c}
x(t) \\
y(t) \\
z(t)
\end{array}\right)+\left(\begin{array}{c}
u(t) \\
v(t) \\
w(t)
\end{array}\right)
$$

der ursprünglichen DGL (2.45) erfolgreich angewandt werden. Man erhält für $\xi^{\prime}$ die DGL

$$
\xi^{\prime}=\frac{1}{2}(q-i p-(u+i v))-\xi(i r+w)+\frac{1}{2} \xi^{2}(q+i p+(u-i v))
$$

und für $\eta^{\prime}$

$$
\eta^{\prime}=\frac{1}{2}(q-i p+(u+i v))-\eta(i r-w)+\frac{1}{2} \eta^{2}(q+i p-(u-i v))
$$

Nach der obigen Rechnung ist das abermalige Auftreten einer RicCATIDGL mit Zusatztermen wenig überraschend.

In der Kinematik ist das beispielsweise der Fall bei den Hüllflächen bei H. R. MüLLER [Mül5.9], dort findet sich auch eine geometrische Interpretation der Abbildungsgleichungen. Die erste Erwähnung eines vergleichbaren Zusammenhanges im Rahmen der NMR stammt von SiLver [S.JH84] man vergleiche auch SiminOvitch [SH.97] und die darin genannten Quellen. 


\section{Drehungen und Kinematik}

\subsection{Ebene Drehungen}

Die Matrix $\boldsymbol{R}$ einer ebenen Drehung um den Winkel $\alpha$ ist in kartesischen Koordinaten gegeben durch

$$
\boldsymbol{R}=\left(\begin{array}{cc}
\cos \alpha & -\sin \alpha \\
\sin \alpha & \cos \alpha
\end{array}\right)
$$

Durch die Gleichungen (미) gewinnt sie die Darstellung

$$
\boldsymbol{R}=\frac{1}{1+t^{2}}\left(\begin{array}{cc}
1-t^{2} & -2 t \\
2 t & 1-t^{2}
\end{array}\right) .
$$

Damit ist eine Drehmatrix durch rationale Funktionen des Tangens des halben Winkels dargestellt.

\subsubsection{Rückgewinnung des Parameters}

Gegeben sei nun eine beliebige Drehmatrix $\boldsymbol{R}$, etwa aus einer Messung von Bahnen abgeleitet. Gesucht ist der zugehörige Parameter $t(\boldsymbol{R})$.

Der antisymmetrische Anteil von $\boldsymbol{R}$ ist $\boldsymbol{R}^{A}:=\frac{1}{2}\left(\boldsymbol{R}-\boldsymbol{R}^{T}\right)$. Er besteht aus der einzigen Komponente

$$
\frac{1}{2}\left(R_{21}-R_{12}\right)=\frac{2 t}{1+t^{2}}=: S
$$

Ferner ist die Spur eine Invariante. Es gilt

$$
\frac{1}{2} \mathrm{Sp} \boldsymbol{R}=\frac{1-t^{2}}{1+t^{2}}=: C
$$

Das Problem der Bestimmung von $t$ aus $S$ und $C$ ist äquivalent zur Inversion von Polarkoordinaten aus Abschnitt 2.7 mit $r=1$. Es gilt also

$$
t=\frac{S}{\sqrt{S^{2}+C^{2}}+C}=\frac{S}{1+C}=\frac{\frac{1}{2}\left(R_{21}-R_{12}\right)}{1+\frac{1}{2} \operatorname{Sp} \boldsymbol{R}}=\frac{R_{21}-R_{12}}{2+\operatorname{Sp} \boldsymbol{R}}
$$




\subsubsection{Zusammensetzung von ebenen Drehungen}

Beginnend mit zwei Matrizen $\boldsymbol{R}_{1}$ und $\boldsymbol{R}_{2}$ vom Typ (․2) berechnet man das Produkt $\boldsymbol{R}_{21}:=\boldsymbol{R}_{2} \boldsymbol{R}_{1}$ und gewinnt daraus mit (इ.5) den Parameter $t_{21}$ der kombinierten Bewegung zurück. Es ergibt sich

$$
t_{21}=\frac{t_{1}+t_{2}}{1-t_{1} t_{2}}
$$

in Übereinstimmung mit dem Additionstheorem (2.3.5) des Tangens.

\subsubsection{Eine Parametrisierung mit Nebenbedingungen}

Durch Einsetzen von $t=s / c$ mit $s:=\sin \left(\frac{\alpha}{2}\right)$ und $c:=\cos \left(\frac{\alpha}{2}\right)$ gewinnt man unter Berücksichtigung von $s^{2}+c^{2}=1$ die Darstellung

$$
\begin{aligned}
\boldsymbol{R} & =\left(\begin{array}{cc}
c^{2}-s^{2} & -2 s c \\
2 s c & c^{2}-s^{2}
\end{array}\right) \\
& =\left(\begin{array}{cc}
2 c^{2}-1 & -2 s c \\
2 s c & 2 c^{2}-1
\end{array}\right) \\
& =\left(\begin{array}{cc}
1-2 s^{2} & -2 s c \\
2 s c & 1-2 s^{2}
\end{array}\right),
\end{aligned}
$$

welche nicht mehr den Grenzübergang $t \rightarrow \pm \infty$ für $c=0$ benötigt. Diese Formeln bekommt man auch direkt unter Benutzung von (ㅍ.3) und (‥4).

Im Gegensatz zum Halbtangens ist die Rückgewinnung der Parameter nicht mehr eindeutig. Durch Vergleich mit der Matrix

$$
R=\left(\begin{array}{cc}
C & -S \\
S & C
\end{array}\right)
$$

(oder wieder durch Benutzung von Spur und antisymmetrischem Anteil) erhält man etwa für die (3.]) die beiden Lösungen

$$
s= \pm \frac{\sqrt{1-C}}{\sqrt{2}} \text { und } c= \pm \frac{S}{\sqrt{2-2 C}} .
$$

Diese Definition ist analog zu den Eulerparametern in drei Dimensionen. Die zwei Parameter $(c, s)$ haben die Nebenbedingung $c^{2}+s^{2}=1$ und sind daher Elemente des Einheitskreises bzw. der $O_{1}$-Gruppe. 


\subsubsection{Eine Matrizenschar}

Wir betrachten nun die Gleichung (B.9) als Definition einer Matrizenschar. Mit den Matrizen

$$
\boldsymbol{I}=\left(\begin{array}{ll}
1 & 0 \\
0 & 1
\end{array}\right) \quad \text { und } \quad \boldsymbol{J}=\left(\begin{array}{cc}
0 & -1 \\
1 & 0
\end{array}\right),
$$

wobei $\boldsymbol{J}^{2}=-\boldsymbol{I}$, schreibt sich die Drehmatrix kurz als $\boldsymbol{R}(\alpha)=\cos (\alpha) \boldsymbol{I}+$ $\sin (\alpha) \boldsymbol{J}$. Wir verallgemeinern dies zu

$$
\boldsymbol{A}:=a \boldsymbol{I}+b \boldsymbol{J}
$$

mit rellen $a$ und $b$. Man liest sofort ab, dass $\operatorname{det} A=a^{2}+b^{2}$ und $\operatorname{tr} A=2 a$. Ferner gilt

$$
\boldsymbol{A}^{-1}=\frac{1}{a^{2}+b^{2}}(a \boldsymbol{I}-b \boldsymbol{J}),
$$

denn es ist

$$
\boldsymbol{A}^{-1} \boldsymbol{A}=\frac{1}{a^{2}+b^{2}}(a \boldsymbol{I}-b \boldsymbol{J})(a \boldsymbol{I}+b \boldsymbol{J})=\frac{a^{2}+b^{2}}{a^{2}+b^{2}} \boldsymbol{I} .
$$

Die Inverse einer Matrix aus der Schar lässt sich damit direkt hinschreiben.

Wir betrachten nun den Spezialfall $a=1$ und die Matrix

$$
\begin{aligned}
(\boldsymbol{I}-b \boldsymbol{J})^{-1}(\boldsymbol{I}+b \boldsymbol{J}) & =\frac{1}{1+b^{2}}(\boldsymbol{I}+b \boldsymbol{J})(\boldsymbol{I}+b \boldsymbol{J}) \\
& =\frac{1-b^{2}}{1+b^{2}} \boldsymbol{I}+\frac{2 b}{1+b^{2}} \boldsymbol{J} .
\end{aligned}
$$

Für $b=\tan (\alpha / 2)$ ist das genau die Darstellung (3.2) einer Drehmatrix. Diese Konstruktion entspricht den CAYLEY-Parametern einer Drehung im Raum.

\subsubsection{Die direkte Ableitung einer Drehmatrix}

Die Matrizenschar des letzten Abschnittes erlaubt die Darstellung

$$
\boldsymbol{R}(t)=\cos (\alpha(t)) \boldsymbol{I}+\sin (\alpha(t)) \boldsymbol{J} .
$$

Diese ist bestens dazu geeignet, Ableitungen nach der Zeit $t$ zu berechnen. 
Mit der Kettenregel folgt

$$
\dot{\boldsymbol{R}(}(t)=(-\sin (\alpha(t)) \boldsymbol{I}+\cos (\alpha(t)) \boldsymbol{J}) \dot{\alpha}(t)=\boldsymbol{J} \boldsymbol{R}(t) \dot{\alpha}(t),
$$

letzteres wegen $\boldsymbol{J}^{2}=-\boldsymbol{I}$. Im Folgenden soll die Abhängigkeit von der Zeit nicht mehr extra notiert werden. Aus (B.J7) folgt direkt $\boldsymbol{J} \boldsymbol{R}=\boldsymbol{R} \boldsymbol{J}$, die Matrizen $\boldsymbol{J}$ und $\boldsymbol{R}$ vertauschen also. Damit bekommt man aus

$$
\dot{\boldsymbol{R}}=\boldsymbol{J} \boldsymbol{R} \dot{\alpha}
$$

die Beziehung

$$
\boldsymbol{J} \dot{\boldsymbol{R}}=\dot{\boldsymbol{R}} \boldsymbol{J}=-\boldsymbol{R} \dot{\alpha} .
$$

Auch die Ableitung von $\boldsymbol{R}$ vertauscht mit $\boldsymbol{J}$.

Durch Differentiation erhält man weiter

$$
\ddot{\boldsymbol{R}}=(\boldsymbol{J} \boldsymbol{R} \dot{\alpha})^{\cdot}=\boldsymbol{J} \dot{\boldsymbol{R}} \dot{\alpha}+\boldsymbol{J} \boldsymbol{R} \ddot{\alpha}=-\boldsymbol{R} \dot{\alpha}^{2}+\boldsymbol{J} \boldsymbol{R} \ddot{\alpha} \quad .
$$

Wählt man insbesondere den Winkel $\alpha$ als Parametrisierung, dann folgt mit $*^{\prime}:=d * / d \alpha$ zunächst $\alpha^{\prime}=1$ und $\alpha^{\prime \prime}=0$, also $\boldsymbol{R}^{\prime \prime}=-\boldsymbol{R}$ und die Ableitungen wiederholen sich. Es gilt dann einfach

$$
\frac{d^{n}}{d \alpha^{n}} \boldsymbol{R}(\alpha)=\frac{d^{n}}{d \alpha^{n}} \cos (\alpha) \boldsymbol{I}+\frac{d^{n}}{d \alpha^{n}} \sin (\alpha) \boldsymbol{J} .
$$

\subsubsection{Die Darstellung im Vierteltangens}

Mit den Einträgen aus der zweiten Zeile der Gleichungstabelle (ए.IU) ergibt sich sofort die Darstellung

$$
\boldsymbol{R}=\frac{1}{\left(1+t^{2}\right)^{2}}\left(\begin{array}{cc}
t^{4}-6 t^{2}+1 & 4 t\left(t^{2}-1\right) \\
-4 t\left(t^{2}-1\right) & t^{4}-6 t^{2}+1
\end{array}\right) \quad .
$$

Fragt man nun nach der Rückgewinnung des Parameters, so erhält man mit

$$
\begin{aligned}
\text { Reduce }\left[\left\{-4 t\left(t^{\wedge} 2-1\right) /\left(1+t^{\wedge} 2\right)^{\wedge} 2\right.\right. & ==s, \\
\left(1-6 t^{\wedge} 2+t^{\wedge} 4\right) /\left(1+t^{\wedge} 2\right)^{\wedge} 2 & ==c\}, t]
\end{aligned}
$$

die Antwort $(s=0 \wedge c=1 \wedge t=0)$ oder für $c \neq 1 \wedge c^{2}+s^{2}=1$

$$
t=\frac{s \pm \sqrt{c^{2}-2 c+s^{2}+1}}{c-1}=\frac{s \pm \sqrt{2-2 c}}{c-1}
$$

welche besagt, dass nun nur noch Drehungen um $(2 n+1) 2 \pi$ von der Inversion ausgenommen sind. Leider ist die übrige Rücktransformation nun nicht mehr eindeutig. 


\subsection{Das Viergelenk}

Das Viergelenk oder auch Gelenkviereck ist ein ebenes Viereck, dessen Ecken man sich durch Scharniere mit Achsen senkrecht zur Zeichenebene gelenkig verbunden denkt s. Abb. B. Es ist technisch wie theoretisch eines der wichtigsten Beispiele der ebenen Kinematik. Als Literatur dazu seien [HI64] oder [LM90] angegeben.

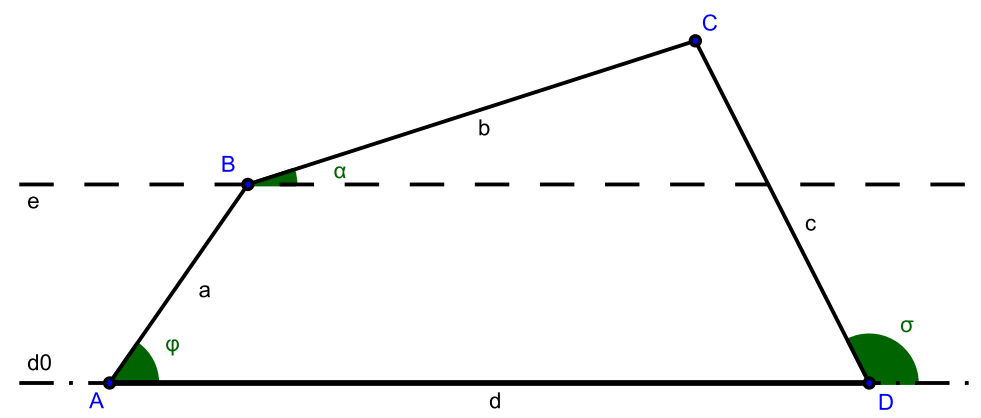

Abbildung 3.1: Ein Viergelenk, hier eine Kurbelschwinge. Die Strecke $\overline{A D}$ wird als befestigt angenommen („Gestell“), die Strecke $\overline{A B}$ dient typischerweise als Antrieb („Pleuel“) und die Strecke $\overline{D C}$ als Abtrieb. Das bewegte System ist mit der Strecke $\overline{B C}$ („Koppel“) verbunden. Die Winkel werden relativ zur Geraden $d_{0}$ gemessen

Die Bedingungen dafür, dass der Streckenzug abcd geschlossen ist, lauten in $x$-Richtung

$$
a \cos \varphi+b \cos \alpha-c \cos \sigma-d=0
$$

und in $y$-Richtung

$$
a \sin \varphi+b \sin \alpha-c \sin \sigma=0 .
$$

Durch Elimination von $\alpha$ mittels $\cos ^{2} \alpha+\sin ^{2} \alpha=1$ erhält man die sog. Freudenstein-Gleichung [Fre.54]

$$
a^{2}+c^{2}+d^{2}+2 c d \cos \sigma=b^{2}+2 a(d \cos \varphi+c \cos (\varphi-\sigma),
$$

welche bei gegebenen Abmessungen eine implizite Gleichung zwischen $\varphi$ und $\sigma$, also zwischen Antriebs- und Abtriebswinkel darstellt. Diese Funktion $\sigma(\varphi)$ nennt man auch Übertragungsfunktion des Viergelenks. 


\section{Drehungen und Kinematik}

Die Gleichung (3.25) ist nur schwer analytisch zu handhaben, weshalb zunächst die Gleichungen (B.2.3) und (B.24) mittels Halbwinkelsubstitution algebraisiert werden, um dann den mittleren Halbwinkel zu eliminieren. Seien $f, t$ und $s$ die zu $\varphi, \alpha$ und $\sigma$ gehörenden Halbwinkel. Dann lauten die Gleichungen für die Geschlossenheit des Streckenzuges

$$
a \frac{1-f^{2}}{1+f^{2}}+b \frac{1-t^{2}}{1+t^{2}}-c \frac{1-s^{2}}{1+s^{2}}-d=0
$$

für die $x$-Richtung und in $y$-Richtung

$$
a \frac{f}{1-f^{2}}+b \frac{t}{1-t^{2}}-c \frac{s}{1-s^{2}}=0 .
$$

Mittels Computeralgebra erhält man daraus durch Elimination von $t$ die algebraische Form der Freudenstein-Gleichung

$$
\begin{aligned}
& \left(1+f^{2}\right)\left((c+d)^{2}+(c-d)^{2} s^{2}+a^{2}\left(1+s^{2}\right)-b^{2}\left(1+s^{2}\right)\right) \\
& \quad=2 a\left(-(c+d)\left(-1+f^{2}\right)+4 c f s+(c-d)\left(-1+f^{2}\right) s^{2}\right)
\end{aligned}
$$

eine algebraische Gleichung vierten Grades, wobei die höchsten einzeln auftretenden Exponenten in $f$ oder $s$ vom Grad zwei sind. Da diese Gleichung also quadratisch in $s$ ist, kann sie mit der Lösungsformel aufgelöst werden. Mit den Abkürzungen

$$
\begin{aligned}
& e=-b^{2}+(a+c-d)^{2}+(a-b-c+d)(a+b-c+d) f^{2} \\
& g=-(b-c)^{2}+(a-d)^{2}+(a+b-c+d)(a-b+c+d) f^{2}( \\
& h=-(b+c)^{2}+(a-d)^{2}+(a-b-c+d)(a+b+c+d) f^{2}(3
\end{aligned}
$$

ergeben sich die beiden Moden des Getriebes zu

$$
s(f)=\frac{4 a c f \pm \sqrt{g h}}{e} .
$$

Identifiziert man nun die Gerade $d_{0}$ aus Abb. 3.] mit der $x$-Achse und den Punkt $A$ mit dem Ursprung, so lauten die Koordinaten der Punkte $B$ und $C$

$$
\overrightarrow{A B}=\left(\begin{array}{c}
\cos \varphi \\
\sin \varphi
\end{array}\right)=\frac{1}{1+f^{2}}\left(\begin{array}{c}
1-f^{2} \\
2 f
\end{array}\right)
$$

und

$$
\overrightarrow{A C}=\left(\begin{array}{c}
d \\
0
\end{array}\right)+\left(\begin{array}{c}
\cos \sigma \\
\sin \sigma
\end{array}\right)=\left(\begin{array}{l}
d \\
0
\end{array}\right)+\frac{1}{1+s^{2}}\left(\begin{array}{c}
1-s^{2} \\
2 s
\end{array}\right)
$$


Daher ist mit den Gleichungen (B.2.9) bis (B.32) eine analytische Beschreibung der Eckpunkte eines Viergelenkes in Abhängigkeit vom Parameter $f$ gefunden, ganz ohne Verwendung trigonometrischer Funktionen.

\subsection{Räumliche Drehungen}

Als Literatur seien hier [Stu64] und [Shu93] angegeben.

Die räumlich geschriebene Erweiterung von (B.2) auf eine Drehung um die $z$-Achse lautet

$$
\boldsymbol{R}=\frac{1}{1+t^{2}}\left(\begin{array}{ccc}
1-t^{2} & -2 t & 0 \\
2 t & 1-t^{2} & 0 \\
0 & 0 & 1+t^{2}
\end{array}\right)
$$

Sei $\boldsymbol{n}$ mit $|\boldsymbol{n}|=1$ die Richtung des Drehvektors, hier der Einheitsvektor in $z$-Richtung, also $\boldsymbol{n}=\boldsymbol{e}_{z}$. Mit den vektorwertigen RodRIGUes-Parametern (=Halbtangens mal Drehrichtung)

$$
t:=t n
$$

wobei $\boldsymbol{t}^{2}=\boldsymbol{t}^{T} \boldsymbol{t}=\boldsymbol{n}^{T} \boldsymbol{n} t^{2}=t^{2}$, lässt sich diese Matrix wie folgt schreiben:

$$
\begin{aligned}
\boldsymbol{R}= & \frac{1}{1+t^{2}}\left(\left(\begin{array}{ccc}
1-t^{2} & 0 & 0 \\
0 & 1-t^{2} & 0 \\
0 & 0 & 1-t^{2}
\end{array}\right)+\left(\begin{array}{ccc}
0 & -2 t & 0 \\
2 t & 0 & 0 \\
0 & 0 & 0
\end{array}\right)\right. \\
& \left.+\left(\begin{array}{ccc}
0 & 0 & 0 \\
0 & 0 & 0 \\
0 & 0 & 2 t^{2}
\end{array}\right)\right) \\
= & \frac{1}{1+\boldsymbol{t}^{2}}\left(\left(1-\boldsymbol{t}^{2}\right) \boldsymbol{I}+2 \boldsymbol{t}^{\times}+2 \boldsymbol{t} \boldsymbol{t}^{T}\right)
\end{aligned}
$$

Hierin ist die Abbildung $\times$ definiert durch

$$
\left(\begin{array}{l}
x \\
y \\
z
\end{array}\right)^{\times}:=\left(\begin{array}{ccc}
0 & -z & y \\
z & 0 & -x \\
-y & x & 0
\end{array}\right) \text {. }
$$

Sie ist die in drei Dimensionen mögliche Zuordnung von einem Vektor zu einer antisymmetrischen Matrix. Insbesondere gilt für jeden Vektor $\boldsymbol{y}$ die Gleichung $\boldsymbol{x}^{\times} \boldsymbol{y}=\boldsymbol{x} \times \boldsymbol{y}$, es handelt sich also um die Darstellung des Kreuzproduktes als Matrixmultiplikation. 
Die Formel (3.37) ist kovariant, d. h. in jeden Bezugssystem richtig. Sie transformiert sich korrekt und ist in einem speziellen Bezugssystem richtig (3.35). Daher kann eine beliebige Drehung im Raum durch eine rationale Funktion eines Vektors dargestellt werden.

Durch einfaches Nachrechnen überzeugt man sich, dass $\boldsymbol{t}$ Eigenvektor zum Eigenwert eins ist.

\subsubsection{Rückgewinnung der Rodrigues-Parameter}

Um aus einer gegebenen Drehmatrix $\boldsymbol{R}$ wieder an $\boldsymbol{t}$ heranzukommen, muss man den gemeinsamen Nenner $1+\boldsymbol{t}^{2}=1+t^{2}$ zunächst isolieren. Aus (3.3.5) erhält man durch Spurbildung

$$
\operatorname{Sp} \boldsymbol{R}=\frac{3-t^{2}}{1+t^{2}}
$$

woraus folgt, dass

$$
1+\operatorname{Sp} \boldsymbol{R}=1+\frac{3-t^{2}}{1+t^{2}}=\frac{1+t^{2}+3-t^{2}}{1+t^{2}}=\frac{4}{1+t^{2}} .
$$

Der antisymmetrische Anteil von (3.37) ist

$$
\boldsymbol{R}^{A}=\frac{1}{2}\left(\boldsymbol{R}-\boldsymbol{R}^{T}\right)=\frac{1}{1+\boldsymbol{t}^{2}} 2 \boldsymbol{t}^{\times},
$$

also gilt für den dualen Vektor mit (3.401)

$$
\frac{2 \boldsymbol{R}^{A \times}}{1+\operatorname{Sp} \boldsymbol{R}}=\frac{2 \cdot 2 \boldsymbol{t} /\left(1+t^{2}\right)}{4 /\left(1+t^{2}\right)}=\boldsymbol{t}
$$

Diese Formel ist kovariant und daher der gesuchte Zusammenhang von Drehmatrix $\boldsymbol{R}$ und den RodRIgues-Parametern $\boldsymbol{t}$.

\subsubsection{Hintereinanderschaltung von 3D-Drehungen}

Wir beginnen mit zwei Drehungen, gegeben durch die Rodrigues-Parameter $\boldsymbol{u}$ und $\boldsymbol{v}$. Die zu $\boldsymbol{u}$ gehörige Drehmatrix lautet also mit (3.37)

$$
\frac{1}{1+\boldsymbol{u}^{2}}\left(\begin{array}{ccc}
u_{x}^{2}-u_{y}^{2}-u_{z}^{2}+1 & 2\left(u_{x} u_{y}-u_{z}\right) & 2\left(u_{y}+u_{x} u_{z}\right) \\
2\left(u_{x} u_{y}+u_{z}\right) & -u_{x}^{2}+u_{y}^{2}-u_{z}^{2}+1 & -2\left(u_{x}-u_{y} u_{z}\right) \\
2\left(u_{x} u_{z}-u_{y}\right) & 2\left(u_{x}+u_{y} u_{z}\right) & -u_{x}^{2}-u_{y}^{2}+u_{z}^{2}+1
\end{array}\right)
$$


Nun bilden wir das Produkt $\boldsymbol{R}(\boldsymbol{t})=\boldsymbol{R}(\boldsymbol{v}) \boldsymbol{R}(\boldsymbol{u})$ und lesen wir mit (B.42) dessen Rodrigues-Parameter $\boldsymbol{t}(\boldsymbol{u}, \boldsymbol{v})$ rückwärts aus. Es ergibt sich mit Computeralgebra in Komponenten

$$
\boldsymbol{t}(\boldsymbol{u}, \boldsymbol{v})=\left(\begin{array}{c}
-\frac{u_{x}+v_{x}+u_{z} v_{y}-u_{y} v_{z}}{u_{x} v_{x}+u_{y} v_{y}+u_{z} v_{z}-1} \\
-\frac{u_{y}-u_{z} v_{x}+v_{y}+u_{x} v_{z}}{u_{x} v_{x}+u_{y} v_{y}+u_{z} v_{z}-1} \\
-\frac{u_{z}+u_{y} v_{x}-u_{x} v_{y}+v_{z}}{u_{x} v_{x}+u_{y} v_{y}+u_{z} v_{z}-1}
\end{array}\right)
$$

oder vektoriell und damit kovariant das Ergebnis

$$
\boldsymbol{t}(\boldsymbol{u}, \boldsymbol{v})=\frac{1}{1-\boldsymbol{u}^{T} \boldsymbol{v}}(\boldsymbol{u}+\boldsymbol{v}-\boldsymbol{u} \times \boldsymbol{v})
$$

\subsubsection{Die Cayleyschen Formeln}

Jeder Drehmatrix $\boldsymbol{R}$ ist über

$$
\boldsymbol{A}:=(\boldsymbol{R}-\boldsymbol{I})(\boldsymbol{R}+\boldsymbol{I})^{-1}
$$

eine antisymmetrische Matrix $\boldsymbol{A}$ zugeordnet, vgl. etwa [Gan.58]. Die Antisymmetrie zeigt man über

$$
\begin{aligned}
\boldsymbol{A}^{T} & =(\boldsymbol{R}+\boldsymbol{I})^{-1 T}(\boldsymbol{R}-\boldsymbol{I})^{T}=\left(\boldsymbol{R}^{T}+\boldsymbol{I}\right)^{-1}\left(\boldsymbol{R}^{T}-\boldsymbol{I}\right) \\
& =\left(\boldsymbol{R}^{T}+\boldsymbol{I}\right)^{-1} \boldsymbol{R}^{-1} \boldsymbol{R}\left(\boldsymbol{R}^{T}-\boldsymbol{I}\right)=(\boldsymbol{I}+\boldsymbol{R})^{-1}(\boldsymbol{I}-\boldsymbol{R})=-\boldsymbol{A} .
\end{aligned}
$$

Die Formel (\$.46) lässt sich nach $\boldsymbol{R}$ auflösen, und es ergibt sich die Darstellung

$$
\boldsymbol{R}=(\boldsymbol{I}-\boldsymbol{A})^{-1}(\boldsymbol{I}+\boldsymbol{A})
$$

für eine Drehmatrix als Funktion der sog. CAYLEY-Parameter.

Durch rückwärts Auslesen mittels Computeralgebra und Formel (B.42) ässt sich zeigen, dass diese genau den Rodrigues-Parametern entsprechen, also dass

$$
A=t^{\times} .
$$

Das lässt sich auch analytisch mit Hilfe der Darstellung (3.37) beweisen. Zunächst ist leicht einzusehen, dass $\boldsymbol{R} \boldsymbol{t}^{\times}=\boldsymbol{t}^{\times} \boldsymbol{R}$. Zu beweisen ist nur die umgestellte Formel (B.47)

$$
\left(I-t^{\times}\right) R=R-t^{\times} R=I+t^{\times} .
$$

Unter Benutzung von

$$
\boldsymbol{t}^{\times 2}=\boldsymbol{t} \boldsymbol{t}^{T}-t^{2} \boldsymbol{I}
$$




\section{Drehungen und Kinematik}

was der Formel für doppelte Kreuzprodukte entspricht, rechnet man nach, dass

$$
\boldsymbol{R} \boldsymbol{t}^{\times}=\boldsymbol{t}^{\times} \boldsymbol{R}=\frac{1}{1+t^{2}}\left(-2 t^{2} \boldsymbol{I}+\left(1-t^{2}\right) \boldsymbol{t}^{\times}+2 \boldsymbol{t} \boldsymbol{t}^{T}\right) .
$$

Die Differenz zur Drehmatrix gewinnt nach Kürzen von $1-t^{2}-\left(-2 t^{2}\right)=$ $1+t^{2}$ die Form

$$
R-R t^{\times}=R-t^{\times} R=I+t^{\times} .
$$

Durch Umstellen bekommt man schließlich

$$
\boldsymbol{R}=\left(\boldsymbol{I}-\boldsymbol{t}^{\times}\right)^{-1}\left(\boldsymbol{I}+\boldsymbol{t}^{\times}\right)=\left(\boldsymbol{I}+\boldsymbol{t}^{\times}\right)\left(\boldsymbol{I}-\boldsymbol{t}^{\times}\right)^{-1},
$$

womit die Gleichheit der CAYLEY- und der Rodrigues-Parameter $\boldsymbol{u}$ und $\boldsymbol{v}$. bewiesen ist, also dass $\boldsymbol{A}=\boldsymbol{t}^{\times}$.

Die Formeln (B.46) und (B.47) sind aber nicht auf drei Dimensionen beschränkt, weshalb man die CAYLEY-Parameter als Verallgemeinerung der RodRIGUEs-Parameter auf beliebig viele Dimensionen auffassen kann.

\subsubsection{Eulerparameter}

Wir benutzen wieder die Abkürzungen $t=s / c$ mit $s:=\sin \left(\frac{\alpha}{2}\right)$ und $c:=\cos \left(\frac{\alpha}{2}\right)$ sowie $\boldsymbol{t}=\boldsymbol{t} \boldsymbol{n}$. Wie in den Gleichung (3.6) bis (3.8) lässt sich die Darstellung (B.37) mit $c^{2}$ erweitern. Dabei beseitigt man gleichzeitig die Singularität bei $\alpha= \pm \pi$ welche dem Grenzübergang $t= \pm \infty$ entpricht. Man erhält

$$
\begin{aligned}
\boldsymbol{R} & =\frac{1}{1+\boldsymbol{t}^{2}}\left(\left(1-\boldsymbol{t}^{2}\right) \boldsymbol{I}+2 \boldsymbol{t}^{\times}+2 \boldsymbol{t} \boldsymbol{t}^{T}\right) \\
& =\frac{1}{c^{2}+s^{2}}\left(\left(c^{2}-s^{2}\right) \boldsymbol{I}+2 c s \boldsymbol{n}^{\times}+2 s^{2} \boldsymbol{n} \boldsymbol{n}^{T}\right) \\
& =\left(2 c^{2}-1\right) \boldsymbol{I}+2 c s \boldsymbol{n}^{\times}+2 s^{2} \boldsymbol{n} \boldsymbol{n}^{T} .
\end{aligned}
$$

Wegen $c^{2}-s^{2}=1-2 s^{2}=2 c^{2}-1$ ist das also eine quadratische Darstellung in den vier Parametern $(c, s \boldsymbol{n})$. Diese ist wegen der Normierungsbedingung ein Element der $\mathrm{O}_{4}$-Drehgruppe. Setzt man also die Parameter in der Form $(c, s \boldsymbol{n})=(a, b, c, d)$ mit der Nebenbedingung $a^{2}+b^{2}+c^{2}+d^{2}=1$ an (dabei bekommt $c$ eine neue Bedeutung), so erhält man aus (3.54) die Darstellung

$$
\boldsymbol{R}=\left(\begin{array}{ccc}
2 a^{2}+2 b^{2}-1 & 2 b c-2 a d & 2 a c+2 b d \\
2 b c+2 a d & 2 a^{2}+2 c^{2}-1 & 2 c d-2 a b \\
2 b d-2 a c & 2 a b+2 c d & 2 a^{2}+2 d^{2}-1
\end{array}\right)
$$


für eine Drehmatrix. Diese vier Parameter heißen EuLERparameter. Ihre Rückgewinnung aus der Matrix beginnt wieder bei der Spur

$$
\mathrm{Sp} \boldsymbol{R}=6 a^{2}+2 b^{2}+2 c^{2}+2 d^{2}-3=4 a^{2}-1,
$$

wobei man sich bei $a$ auf ein Vorzeichen (Winkelbereich) festzulegen hat. Den Vektorteil bekommt man für $a \neq 0$, wie bei den RodriguesParametern, über den antisymmetrischen Anteil

$$
R^{\times}=\left(\begin{array}{c}
2 a b \\
2 a c \\
2 a d
\end{array}\right) .
$$

Für $a=0$ benutzt man die Spurelemente und den symmetrischen Anteil.

\subsubsection{Eulerparameter hintereinandergeschalteter Drehungen}

Durch Rückgewinnung der EulERparameter weist man nach (am besten mit Hilfe von Computeralgebra), dass das Produkt zweier Drehmatrizen $\boldsymbol{R}_{21}=\boldsymbol{R}_{2} \boldsymbol{R}_{1}$ auf die Parameter

$$
\begin{aligned}
a_{21} & = \pm\left(a_{1} a_{2}-b_{1} b_{2}-c_{1} c_{2}-d_{1} d_{2}\right) \\
b_{21} & =a_{2} b_{1}+a_{1} b_{2}+c_{2} d_{1}-c_{1} d_{2} \\
c_{21} & =a_{2} c_{1}+a_{1} c_{2}-b_{2} d_{1}+b_{1} d_{2} \\
d_{21} & =b_{2} c_{1}-b_{1} c_{2}+a_{2} d_{1}+a_{1} d_{2}
\end{aligned}
$$

führt. Das lässt sich für den Fall "+" als Produkt von Matrizen des Typs

$$
\boldsymbol{Q}=\left(\begin{array}{cccc}
a & b & c & d \\
-b & a & -d & c \\
-c & d & a & -b \\
-d & -c & b & a
\end{array}\right)=a \boldsymbol{I}+b \boldsymbol{Q}_{b}+c \boldsymbol{Q}_{c}+d \boldsymbol{Q}_{d}
$$

schreiben, wobei die Einträge in den Basismatrizen nur aus $\{0,1,-1\}$ bestehen. Damit ist eine multiplikative Darstellung der Drehgruppe in EuLERparmetern gefunden.

Die Basismatrizen bilden eine Gruppe mit folgender Multiplikationstafel $\left(\boldsymbol{I} \equiv 1, \boldsymbol{Q}_{b} \equiv i, \boldsymbol{Q}_{c} \equiv j, \boldsymbol{Q}_{d} \equiv k\right)$

\begin{tabular}{c|cccc} 
& 1 & $i$ & $j$ & $k$ \\
\hline 1 & 1 & $i$ & $j$ & $k$ \\
$i$ & $i$ & -1 & $k$ & $-j$ \\
$j$ & $j$ & $-k$ & -1 & $i$ \\
$k$ & $k$ & $j$ & $-i$ & -1
\end{tabular}


Dies ist die Multiplikationstabelle für Quaternionen. EuLERparameter sind also Quaternionen.

\subsubsection{Die Ableitung einer Drehmatrix}

Die Matrix $\boldsymbol{R}$ möge von der Zeit $t$ abhängen, also $\boldsymbol{R}(t)$. Aus

$$
\mathbf{0}=\partial_{t} \boldsymbol{I}=\partial_{t}\left(\boldsymbol{R} \boldsymbol{R}^{T}\right)=\dot{\boldsymbol{R}} \boldsymbol{R}^{T}+\boldsymbol{R} \dot{\boldsymbol{R}}^{T}=\dot{\boldsymbol{R}} \boldsymbol{R}^{T}+\left(\dot{\boldsymbol{R}} \boldsymbol{R}^{T}\right)^{T}
$$

folgt die Antisymmetrie von $\dot{\boldsymbol{R}} \boldsymbol{R}^{T}:=\boldsymbol{\Omega}$. Ebenso folgt aus $\mathbf{0}=\partial_{t}\left(\boldsymbol{R}^{T} \boldsymbol{R}\right)$ die Antisymmetrie von $\boldsymbol{R}^{T} \dot{\boldsymbol{R}}$.

Interpretiert man nun die Gleichung

$$
\dot{R}=\Omega R
$$

als Differentialgleichung mit konstanten Koeffizienten, so erhält man als formale Lösung

$$
\boldsymbol{R}(t)=\exp (\boldsymbol{\Omega} \cdot t)
$$

mit der Exponentialfunktion für Matrizen. Hierin versteht man die innere Ableitung $\boldsymbol{\Omega}$ als der Drehung $\boldsymbol{R}$ nachgeschaltet.

Setzt man $\boldsymbol{\Omega}=\boldsymbol{\omega}^{\times}$, so gilt für einen Vektor $\boldsymbol{r}$ die Gleichung $\boldsymbol{\Omega} \boldsymbol{r}=$ $\boldsymbol{\omega} \times \boldsymbol{r}$. Der axiale Vektor $\boldsymbol{\omega}$ ist also der Darbouxsche Drehvektor einer infinitesimalen Drehung mit Winkel $|\boldsymbol{\omega}|$.

Analog hat jede Differentialgleichung des Typs

$$
\dot{r}=\omega \times r
$$

mit räumlichen Drehungen zu tun. Eine solche Drehung wird aber nach den Überlegungen aus Abschnitt 3.31 durch die Parametrisierung durch die Rodrigues-CAYley-Parameter

$$
\boldsymbol{t}=\frac{\boldsymbol{\omega}}{|\boldsymbol{\omega}|} \tan \left(\frac{|\boldsymbol{\omega}|}{2}\right)
$$

besonders einfach, und auch die Zeitentwicklung ist gutartig, denn sie transformiert sich nach Abschnitt 2 rational. Folglich ist die Halbwinkelsubstitution beim Auffinden und Interpretieren der Lösung einer solchen Differentialgleichung nützlich, ganz besonders im Hinblick auf Computeralgebra. 


\subsection{Drehungen als komplexe Zahlen und Matrizen}

Wir betrachten noch einmal die Matrizenschar $\boldsymbol{A}=a \boldsymbol{I}+b \boldsymbol{J}$ aus Kapitel [..4. Das Produkt zweier Matrizen ist dann gegeben als

$$
\boldsymbol{A}_{21}=\left(a_{2} \boldsymbol{I}+b_{2} \boldsymbol{J}\right)\left(a_{1} \boldsymbol{I}+b_{1} \boldsymbol{J}\right)=\left(a_{2} a_{1}-b_{2} b_{1}\right) \boldsymbol{I}+\left(b_{2} a_{1}+a_{2} b_{1}\right) \boldsymbol{J} .
$$

Dies entspricht der Multiplikationregel für komplexe Zahlen. Also gilt die Isomorphie

$$
a \boldsymbol{I}+b \boldsymbol{J} \cong a+b i
$$

leicht zu merken mit $\boldsymbol{J}^{2}=-\boldsymbol{I}$. Eine Drehung $\boldsymbol{R}=\cos (\alpha) \boldsymbol{I}+\sin (\alpha) \boldsymbol{J}$ entspricht dann mit der MoIvREschen Formel der komplexen Zahl

$$
z=\cos (\alpha)+i \sin (\alpha)=\exp (i \alpha)
$$

$\operatorname{mit}|z|=1$.

Kommen wir nun zurück auf die Matrizendarstellung (B.60) der Quaternionen. Teilt man die 4x4-Matrix in vier 2x2-Blöcke auf, so gilt

$$
\boldsymbol{Q}=\left(\begin{array}{cc|cc}
a & b & c & d \\
-b & a & -d & c \\
\hline-c & d & a & -b \\
-d & -c & b & a
\end{array}\right)=\left(\begin{array}{c|c}
a \boldsymbol{I}-b \boldsymbol{J} & c \boldsymbol{I}-d \boldsymbol{J} \\
\hline-c \boldsymbol{I}-d \boldsymbol{J} & a \boldsymbol{I}+b \boldsymbol{J}
\end{array}\right)
$$

und das ist wegen (3.6.5) isomorph zur komplexen 2x2-Matrix

$$
\begin{aligned}
\boldsymbol{Q} & \cong\left(\begin{array}{cc}
a-i b & c-i d \\
-c-i d & a+i b
\end{array}\right) \\
& =a\left(\begin{array}{ll}
1 & 0 \\
0 & 1
\end{array}\right)+b\left(\begin{array}{cc}
-i & 0 \\
0 & i
\end{array}\right)+c\left(\begin{array}{cc}
0 & 1 \\
-1 & 0
\end{array}\right)+d\left(\begin{array}{cc}
0 & -i \\
-i & 0
\end{array}\right)
\end{aligned}
$$

Die Matrix (3.66) ist die CAYley-KLein-Darstellung einer Drehmatrix. Multipliziert man die letzten drei Matrizen der unteren Zeile mit $i$, so entstehen die PAULI-Matrizen

$$
\left(\begin{array}{cc}
1 & 0 \\
0 & -1
\end{array}\right),\left(\begin{array}{cc}
0 & i \\
-i & 0
\end{array}\right) \text { und }\left(\begin{array}{ll}
0 & 1 \\
1 & 0
\end{array}\right) \text {. }
$$

Gegenüber der Lehrbuchnotation ist die Reihenfolge und damit ein Vorzeichen vertauscht. 


\subsection{Instantane Kinematik}

Die Kinematik ist die Lehre der Bewegungen ohne Berücksichtigung der Kräfte, also ein Teilgebiet der Mechanik. Gegeben sei eine zwangläufige Bewegung mit $\boldsymbol{R}(t)$ Drehmatrix und $\boldsymbol{d}(t)$ Verschiebevektor.

Die Bahn $\boldsymbol{x}(t)$ (auch Spur, Trajektorie, Koppelkurve oder Orbit genannt) im Laborsystem (auch Rastsystem genannt) eines Punktes $\boldsymbol{\xi}$ des starren Körpers mit Koordinaten im körperfesten System (auch Gangsystem genannt) ist

$$
\boldsymbol{x}(t)=\boldsymbol{R}(t) \boldsymbol{\xi}+\boldsymbol{d}(t) .
$$

Dies ist lineare Funktion in $\boldsymbol{\xi}$, welche als zeitabhängige Transformation zwischen Rastsystem und Gangsystem verstanden werden kann.

Die Ableitung dieser Transformation nach der Zeit ergibt

$$
\dot{\boldsymbol{x}}(t)=\dot{\boldsymbol{R}}(t) \boldsymbol{\xi}+\dot{\boldsymbol{d}}(t)
$$

und nach Einsetzen von (3.67) folgt

$$
\dot{\boldsymbol{x}}=\dot{\boldsymbol{R}}\left(\boldsymbol{R}^{T}(\boldsymbol{x}-\boldsymbol{d})\right)+\dot{\boldsymbol{d}}=\boldsymbol{\Omega}(\boldsymbol{x}-\boldsymbol{d})+\dot{\boldsymbol{d}} .
$$

Umsortieren der Terme liefert

$$
\dot{x}-\Omega x=\dot{d}-\Omega d=: v
$$

Dieser Ausdruck hängt nicht von der Wahl des Bezugspunktes $\boldsymbol{\xi}$ ab und ist damit eine sog. kinematische Invariante). Er besitzt eine vage Interpretation als abstrakte Geschwindigkeit des starren Körpers. Ich möchte im Folgenden den Namen „Systemgeschwindigkeit" verwenden.

Sei nun $\boldsymbol{\omega}^{\times}=\boldsymbol{\Omega}$ die zugehörige Winkelgeschwindigkeit. Für jede Bahn eines Punktes des starren Körpers gilt also die Beziehung

$$
\dot{\boldsymbol{x}}=\boldsymbol{\omega} \times \boldsymbol{x}+\boldsymbol{v},
$$

bekannt als Euler-Gleichung. Das Paar $(\boldsymbol{\omega}, \boldsymbol{v})$ nennt man Kinemate. Man kann die Gleichung (3.T) als inhomogene Variante der Gleichung (‥63) auffassen. Beide Gleichungen lassen sich auf zwei entkoppelte RICCATI-Differentialgleichungen reduzieren, vgl. Abschnitt 2.J.

Diese Formel besitzt eine geometrische Interpretation. Zunächst spaltet man die Systemgeschwindigkeit $\boldsymbol{v}$ in Anteile senkrecht und parallel zu $\boldsymbol{\omega}$ 
auf, also $\boldsymbol{v}=\boldsymbol{v}^{\|}+\boldsymbol{v}^{\perp}$. Mit

$$
\begin{aligned}
\omega & :=|\boldsymbol{\omega}| \\
\boldsymbol{r} & :=\boldsymbol{\omega} / \omega \\
\boldsymbol{a} & :=\frac{\boldsymbol{\omega} \times \boldsymbol{v}}{\omega^{2}} \\
\tau & :=\frac{\boldsymbol{\omega} \cdot \boldsymbol{v}}{\omega^{2}}
\end{aligned}
$$

gilt

$$
\boldsymbol{v}^{\perp}=\boldsymbol{\omega} \times(-\boldsymbol{a}) \quad \text { und } \quad \boldsymbol{v}^{\|}=\omega \tau \boldsymbol{r}
$$

und die EulER-Gleichung erhält die Form

$$
\dot{\boldsymbol{x}}=\boldsymbol{\omega} \times \boldsymbol{x}+\boldsymbol{v}=\omega \boldsymbol{r} \times \boldsymbol{x}+\boldsymbol{v}^{\perp}+\boldsymbol{v}^{\|}=\omega(\boldsymbol{r} \times(\boldsymbol{x}-\boldsymbol{a})+\tau \boldsymbol{r}) .
$$

Es findet also eine Drehung um eine Gerade mit Aufpunkt $\boldsymbol{a}$ und Richtung $\boldsymbol{r}$ bei gleichzeitiger paralleler Verschiebung entlang dieser Geraden statt. Eine solche Bewegung nennt man Schraubung. Die Größen (B.r2) bis (3.r.5), von denen wegen $\boldsymbol{r}^{T} \boldsymbol{r}=1$ und $\boldsymbol{r}^{T} \boldsymbol{a}=0$ nur sechs unabhängig sind, nennt man Schraubparameter. In diesen neuen Größen lauten die RodRigues-CAYley Parameter

$$
\boldsymbol{t}=\boldsymbol{r} \tan \left(\frac{\omega}{2}\right)
$$

Die Gleichung (B.T]) lässt sich auch homogen als einzelne Matrixgleichung schreiben:

$$
\frac{\partial}{\partial t}\left(\begin{array}{c}
\boldsymbol{x} \\
1
\end{array}\right)=\left(\begin{array}{cc}
\boldsymbol{\Omega} & \boldsymbol{v} \\
\mathbf{0}^{T} & 0
\end{array}\right)\left(\begin{array}{c}
\boldsymbol{x} \\
1
\end{array}\right) .
$$

Insgesamt zeigen die Überlegungen, dass die Halbwinkelsubstitution auch in der Kinematik des starren Körpers nützlich sein könnte. 



\section{Anwendungen in der Physik}

In diesem Kapitel sollen diejenigen Anwendungen in der Physik besprochen werden, die zu den Überlegungen dieses Buches führten. Nach den Kapiteln über Anwendungen in Mathematik und Kinematik beschließt dieser Abschnitt das Büchlein.

Neben allgemeinen Zusammenhängen für die Verteilungsfunktionen entstammen die Beispiele überwiegend der Kernspinresonanz. Die grundlegende Gleichung ist die BLOCH-Gleichung, welche eine Verwandtschaft zur Euler-Gleichung der Kinematik aufweist. Die Halbwinkelsubstitution findet eben bei allen Phänomenen Anwendung, die mit Drehungen zu tun haben, ob im Ortsraum oder dem Raum der Magnetisierung. Ferner wird sich die hyperbolische Substitution als nützlich erweisen.

Es wären sicher noch Beispiele für die optischen BLOCH-Gleichungen zu erwarten. Auf diesem Gebiete bin ich aber leider zu wenig bewandert, um einen Beitrag leisten zu können. Die bloße Erwähnung muss hier genügen.

\subsection{Verteilungsfunktionen}

Die den makroskopischen Größen zu Grunde liegende Funktion ist die Verteilungsfunktion. Im Unterschied zur klassischen MAXwELL-BOLTZMANN Verteilung hat man in der Quantentenstatistik für halbzahligen Spin die Fermi-Dirac (FD) und für ganzzahligen Spin die Bose-Einstein (BE) Statistik. Mit der Zuordnung $\mathrm{FD}=$ "+" und $\mathrm{BE}=$-" erhält man die Verteilungsfunktionen

$$
f(E)=\frac{1}{e^{\frac{E-\mu}{k_{B} T}} \pm 1}=\frac{1}{e^{x} \pm 1}
$$

wobei $E$ die Energie, $\mu$ das chemische Potential, $k_{B}$ die Boltzmann-Konstante, $T$ die Temperatur und $x=\frac{E-\mu}{k_{B} T}$ ist. Sei nun $h=\tanh \frac{x}{2}$. Dann 
gilt mit (1.25)

$$
\begin{aligned}
f(h) & =\frac{1}{\frac{1+h}{1-h} \pm 1}=\frac{1-h}{1+h \pm(1-h)} \\
& = \begin{cases}\frac{1-h}{2}= & \frac{1}{2}\left(1-\tanh \frac{x}{2}\right) \\
\frac{1-h}{2 h}=\frac{1}{2}\left(\frac{1}{h}-1\right)=\frac{1}{2}\left(\operatorname{coth} \frac{x}{2}-1\right) & (\mathrm{BE})\end{cases}
\end{aligned}
$$

Tatsächlich lassen sich also die grundlegenden Verteilungsfunktionen in einfacher Weise durch den Halbtangenshyperbolikus ausdrücken.

\subsection{Die Besetzungszahldifferenz}

Betrachtet wird ein System aus zwei nicht wechselwirkenden Quantenzuständen (z.B. Spins) mit den Teilchenzahlen $N_{+}$und $N_{-}$und der Gesamtzahl $N=N_{+}+N_{-}$. Im thermischen Gleichgewicht gilt für genügend hohe Temperaturen die Boltzmann-Verteilung

$$
N_{+}=N_{-} \exp \left(-\frac{\Delta E}{k T}\right) .
$$

Die Besetzungszahldifferenz ist nun einfach

$$
\begin{aligned}
\frac{\Delta N}{N} & =\frac{N_{-}-N_{+}}{N_{-}+N_{+}}=\frac{N_{-}\left(1-\exp \left(-\frac{\Delta E}{k T}\right)\right)}{N_{-}\left(1+\exp \left(-\frac{\Delta E}{k T}\right)\right)} \\
& =\frac{\exp \left(+\frac{\Delta E}{2 k T}\right)-\exp \left(-\frac{\Delta E}{2 k T}\right)}{\exp \left(+\frac{\Delta E}{2 k T}\right)+\exp \left(-\frac{\Delta E}{2 k T}\right)}=\tanh \frac{\Delta E}{2 k T} .
\end{aligned}
$$

Man bekommt hier den tanh durch die Herleitung geschenkt. In Hochtemperaturnäherung ist das Argument klein und es gilt daher

$$
\frac{\Delta N}{N}=\frac{\Delta E}{2 k T} \text {. }
$$

Aus den Zeeman-Energieniveaus von Elektronenspins im Magnetfeld $E=$ $\pm \frac{1}{2} g \mu_{B} B_{0}$ ( $\mu_{B}$ BoHRsches Magneton, $g$ LANDÉ-Faktor) resultiert der Energieabstand $\Delta E=g \mu_{B} B_{0}$. Analog gilt mit dem Kerndipolmoment $\mu_{K}$ für die Kernspinresonanz

$$
\frac{\Delta N}{N}=\frac{\mu_{K} B_{0}}{2 k T}
$$

also $\Delta N \sim B_{0} / T$. Die Magnetisierung des Kernspins im äußeren Magnetfeld ist ein Beispiel einer solchen Hochtemperaturnäherung. 


\subsection{Die Bloch-Gleichung}

Die BLOCH-Gleichung [Blo46] für die Magnetisierung $\boldsymbol{M}$ und die magnetische Feldstärke $\boldsymbol{B}$ ist vom Typ $\left(\gamma=\mu_{K} / \hbar\right.$ gyromagnetisches Verhältnis) lautet

$$
\dot{M}=-\gamma \boldsymbol{B} \times \boldsymbol{M}-\boldsymbol{D} \boldsymbol{M}+\boldsymbol{p} .
$$

Hierin ist $\boldsymbol{B}=\boldsymbol{B}_{0}+\boldsymbol{B}_{1}$ mit $\left|\boldsymbol{B}_{1}\right| \ll\left|\boldsymbol{B}_{0}\right|$, wobei konventionsgemäß

$$
\boldsymbol{B}_{0}=\left(\begin{array}{c}
0 \\
0 \\
B_{0}
\end{array}\right) \quad \text { und } \quad \boldsymbol{B}_{1}=\left(\begin{array}{c}
B_{1 x}(t) \\
B_{1 y}(t) \\
0
\end{array}\right)
$$

mit der in den Raten $r_{1}:=1 / T_{1}$ und $r_{2}:=1 / T_{2}$ geschriebenen Diagonalmatrix (Dämpfung, Relaxation)

$$
\boldsymbol{D}:=\left(\begin{array}{ccc}
r_{2} & 0 & 0 \\
0 & r_{2} & 0 \\
0 & 0 & r_{1}
\end{array}\right)
$$

und der Inhomogenität $\boldsymbol{p}$

$$
\boldsymbol{p}=\left(\begin{array}{c}
0 \\
0 \\
r_{1} M_{0}
\end{array}\right),
$$

worin $M_{0}$ die Gleichgewichtsmagnetisierung ist. Diese Gleichung stellt eine Verallgemeinerung der in Abschnitt 2.1 besprochenen DGL dar, da sie zusätzlich einen linearen Dämpfungsterm besitzt.

Die homogene BLOCH-Gleichung ist vom Typ

$$
\dot{M}=-\gamma \boldsymbol{B} \times \boldsymbol{M}
$$

Hierin übernimmt der Term $-\gamma \boldsymbol{B}=: \boldsymbol{h}$ die Rolle des Drehvektors in Formel (3.6.3). Dieser Gleichungstyp findet als optische Bloch-Gleichung in der Quantenoptik Anwendung, vgl. etwa FeYNMAN et al. [FV.JH57].

\subsubsection{Freie Präzession}

Sei $\boldsymbol{B}=\left(0,0, B_{0}\right)^{T}$ ein Magnetfeld in $z$-Richtung und die Magnetisierung gegeben in den Komponenten $\boldsymbol{M}=\left(M_{x}, M_{y}, M_{z}\right)^{T}$. Dann ist die Lösung 


\section{Anwendungen in der Physik}

von (4.6) einfach

$$
\left(\begin{array}{c}
M_{x} \\
M_{y} \\
M_{z}
\end{array}\right)=\left(\begin{array}{c}
c_{2} \sin \left(B_{0} \gamma t\right)+c_{1} \cos \left(B_{0} \gamma t\right) \\
c_{2} \cos \left(B_{0} \gamma t\right)-c_{1} \sin \left(B_{0} \gamma t\right) \\
c_{3}
\end{array}\right)
$$

und beschreibt die freie Präzession der Magnetisierung in der $x y$-Ebene. Die drei Integrationskonstanten $c_{i}$ sind aus den Anfangsbedingungen zu bestimmen. Sie haben die Einheit einer Magnetisierung und werden typischerweise als Vielfache von $M_{0}$ ausgedrückt. Der Ausdruck $\omega_{0}:=\gamma B_{0}$ ist die LARMOR-Frequenz.

\subsubsection{Mit Dämpfung (Relaxation)}

Man beschreibt den Prozess der Relaxation durch Hinzunahme eines Dämpfungstermes, mathematisch gesehen einer Inhomogenität. Zuständig ist dann die inhomogene Bloch-Gleichung (4.2). Das Abklingverhalten bei einem statischen $\boldsymbol{B}$-Feld wird beschrieben durch

$$
\left(\begin{array}{c}
M_{x} \\
M_{y} \\
M_{z}
\end{array}\right)=\left(\begin{array}{c}
c_{2} e^{-r_{2} t} \sin \left(B_{0} \gamma t\right)+c_{1} e^{-r_{2} t} \cos \left(B_{0} \gamma t\right) \\
c_{2} e^{-r_{2} t} \cos \left(B_{0} \gamma t\right)-c_{1} e^{-r_{2} t} \sin \left(B_{0} \gamma t\right) \\
c_{3} e^{-r_{1} t}+M_{0}
\end{array}\right),
$$

also eine exponentiell gedämpfte Präzession, bekannt unter dem Namen Free Induction Decay (FID).

\subsubsection{Erzwungene Schwingungen}

Die Idee ist es, die gedämpfte Präzession durch ein rotierendes Wechselfeld der Frequenz $\omega$ experimentell anzuregen. Das zu realisierende magnetische Feld ist demnach (bis auf einen konstanten Phasen-Offset)

$$
\boldsymbol{B}=\left(\begin{array}{c}
B_{1} \cos (\omega t) \\
-B_{1} \sin (\omega t) \\
B_{0}
\end{array}\right)
$$

mit $B_{1} \ll B_{0}$. Zur Lösung macht man den mathematischen Ansatz einer mit der Anregungsfrequenz rotierenden Magnetisierung (Variation der Konstanten der Lösung (4.7))

$$
\left(\begin{array}{c}
M_{x} \\
M_{y} \\
M_{z}
\end{array}\right)=\left(\begin{array}{c}
m_{x}(t) \cos (\omega t)+m_{y}(t) \sin (\omega t) \\
m_{y}(t) \cos (\omega t)-m_{x}(t) \sin (\omega t) \\
m_{z}(t)
\end{array}\right) .
$$


Mit diesem Ansatz, der einem rotierenden Koordinatensystem entspricht, und der Abkürzung $\omega_{0}:=\gamma B_{0}$ vereinfacht sich die DGL in $\boldsymbol{m}$ zu

$$
\begin{aligned}
\left(\begin{array}{c}
\dot{m}_{x}(t) \\
\dot{m}_{y}(t) \\
\dot{m}_{z}(t)
\end{array}\right) & =\left(\begin{array}{c}
m_{y}(t)\left(\omega_{0}-\omega\right)-r_{2} m_{x}(t) \\
B_{1} \gamma m_{z}(t)+m_{x}(t)\left(\omega-\omega_{0}\right)-r_{2} m_{y}(t) \\
r_{1}\left(M_{0}-m_{z}(t)\right)-B_{1} \gamma m_{y}(t)
\end{array}\right) \\
& =\left(\begin{array}{ccc}
-r_{2} & -\Delta \omega & 0 \\
\Delta \omega & -r_{2} & \gamma B_{1} \\
0 & -\gamma B_{1} & -r_{1}
\end{array}\right)\left(\begin{array}{c}
m_{x} \\
m_{y} \\
m_{z}
\end{array}\right)+\left(\begin{array}{c}
0 \\
0 \\
r_{1} M_{0}
\end{array}\right)
\end{aligned}
$$

wobei $\Delta \omega:=\omega-\omega_{0}$.

\subsubsection{Stationäre Lösungen}

Falls die Resonanzkurve experimentell genügend langsam abgetastet wird, verschwinden die Ableitungen von (4.8) und man erhält die stationären Lösungen

$$
\left(\begin{array}{l}
m_{x}(t) \\
m_{y}(t) \\
m_{z}(t)
\end{array}\right)=\left(\begin{array}{c}
-\frac{B_{1} \gamma M_{0} r_{1}\left(\omega-\omega_{0}\right)}{B_{1}^{2} \gamma^{2} r_{2}+r_{1}\left(r_{2}^{2}+\left(\omega-\omega_{0}\right)^{2}\right)} \\
\frac{B_{1} \gamma M_{0} r_{1} r_{2}}{B_{1}^{2} \gamma^{2} r_{2}+r_{1}\left(r_{2}^{2}+\left(\omega-\omega_{0}\right)^{2}\right)} \\
\frac{M_{0} r_{1}\left(r_{2}^{2}+\left(\omega-\omega_{0}\right)^{2}\right)}{B_{1}{ }^{2} \gamma^{2} r_{2}+r_{1}\left(r_{2}{ }^{2}+\left(\omega-\omega_{0}\right)^{2}\right)}
\end{array}\right) .
$$

Die rotierende Magnetisierung im Laborsystem ergibt sich nun durch Rücktransformation der Gleichungen.

\subsubsection{Die Riccati-Gleichung und Pulse}

Diese Überlegungen fanden zuerst Eingang in die Kernspinresonanz durch Silver et al. [S.JH84]. Man vergleiche auch das Kapitel [.].

Für einen Puls mit einer Dauer deutlich unterhalb der Relaxationszeiten kann man die Dämpfungsterme $r_{1}$ und $r_{2}$ in der "Rotating Wave Approximation" (4.8) streichen. Zusätzlich hat man ein $B$-Feld in $y$-Richtung einzuführen und erhält die DGL

$$
\left(\begin{array}{c}
\dot{m}_{x}(t) \\
\dot{m}_{y}(t) \\
\dot{m}_{z}(t)
\end{array}\right)=\left(\begin{array}{ccc}
0 & -\Delta \omega & -\gamma B_{1 y}(t) \\
\Delta \omega & 0 & \gamma B_{1 x}(t) \\
\gamma B_{1 y}(t) & -\gamma B_{1 x}(t) & 0
\end{array}\right)\left(\begin{array}{c}
m_{x} \\
m_{y} \\
m_{z}
\end{array}\right) \text {. }
$$




\section{Anwendungen in der Physik}

Mit den komplexen Magnetisierung $m:=m_{x}+i m_{y}$ und der komplexen Kreisfrequenz $\Omega:=\gamma\left(B_{1 x}+i B_{1 y}\right)$ erhält man die komplexen DGLn

$$
\begin{aligned}
& 0=\dot{m}+i \Delta \omega m+i \Omega m_{z} \\
& 0=\dot{m}_{z}+\frac{i}{2}(\bar{\Omega} m-\Omega \bar{m}),
\end{aligned}
$$

wobei $\bar{*}$ das konjugiert-Komplexe von $*$ ist. Mittels der stereografischen Projektion führen wir nun ein

$$
f:=\frac{m}{m_{0}+m_{z}},
$$

wobei $m_{0}=\sqrt{m_{x}^{2}+m_{y}^{2}+m_{z}^{2}}$. Durch Differentiation von $f$ nach der Zeit erhalten wir unter Benutzung der komplexen DGLn (4.9) und (4.JU) die BLOCH-RICCATI-DGL

$$
0=\dot{f}+i \Delta \omega f-\frac{i}{2} \bar{\Omega} f^{2}+i \frac{\Omega}{2}
$$

Die Abbildung 2.4 (auf den Kopf gestellt, der Pol der stereografischen Projektion liegt im Südpol) enthüllt nun eine grundlegende Bedeutung der neuen Funktion $f$ : Ist der momentane Flipwinkel ${ }^{\mathbb{W}}$ gegeben durch $\sin \alpha:=|m| / m_{0}$ so ist der Betrag

$$
|f|=\frac{\sin \alpha}{1+\cos \alpha}=\tan \left(\frac{\alpha}{2}\right)
$$

genau unsere Halbwinkelsubstitution.

\subsection{Die Flash-Sequenz}

Die FLASH ${ }^{\mathbb{Z}_{-}}$Sequenz wurde eingeführt von HAASE et al. [ $\left.\mathrm{HFM}^{+} 85\right]$. Sie ist eine Messvorschrift mit einer komplexen Folge spezieller Pulse, bei der vorwiegend die längs zur Hauptfeldrichtung gelegene Magnetisierung genutzt wird. Ich folge hier den Arbeiten [HDD(08], [DH10] und [HDWD11], welche die Signalgleichung diskutieren. Diese beschreibt die Abhängigkeit

\footnotetext{
${ }^{1}$ Der Flipwinkel, auch Kippwinkel genannt, beschreibt die Auslenkung der Magnetisierung von der Richtung des Hauptfeldes nach Ende eines Hochfrequenzpulses, kurz HF-Puls genannt. Er ist damit ein experimentell einstellbarer Parameter.

${ }^{2}$ Abkürzung für Fast Low Angle SHot. Andere Bezeichnungen sind SPGR (Spoiled Gradient Echo) oder FFE (Fast Field Echo).
} 
der gemssenen Amplitude als Funktion der Messgrößen (Längsrelaxationszeit $T_{1}$ und Querrelaxationszeit $T_{2}$ ) und der experimentell einstellbaren Parameter. Bezüglich der verwendeten Terminologie sei hiermit abermals auf das im Internet verfügbare umfangreiche MR-Glossar der Firma Siemens verwiesen.

In der Medizin ist die Messdauer entscheidend: Je kürzer, desto besser. Wichtige Sequenzen der bildgebenden Verfahren der Kernspintomografie (wie die Flash-Sequenz) sind somit auf schnelle Bildgebung angelegt. Das bedeutet in der Praxis, dass

- die Repetitionszeit $T_{R}$ zwischen den Hochfrequenzpulsen kleiner als die Relaxationszeiten $T_{1}$ und $T_{2}$ ist,

- die Sequenzen mit kleinen Flipwinkeln arbeiten.

Die Gleichung für die Signalstärke $S$ einer Flash-Sequenz ist

$$
S=\frac{A \sin (\alpha)\left(1-e^{-\frac{T_{R}}{T_{1}}}\right)}{1-\cos (\alpha) e^{-\frac{T_{R}}{T_{1}}}},
$$

wobei $T_{R}$ die Repetitionszeit der HF-Pulse, $\alpha$ der Flipwinkel und $A \sim$ $M_{0} \exp \left(-T_{E} / T_{2}^{*}\right)$ die Amplitude in arbiträren Einheiten ist. ${ }^{[}$

Die Argumente der Exponentialfunktion sind damit negativ und klein. Wegen $\exp (-x) \approx 1-x$ sind die Werte daher kleiner als Eins. Es ist daher ratsam, die Substitutionen für kleine Argumente aus Abschnitt [.6] anzuwenden.

Die manuelle Durchführung der Rechnung ist sehr instruktiv, weshalb noch einmal auf [DH10] hingewiesen werden soll. Die Substitution mit Mathematica

$$
\text { St=Simplify }[\operatorname{TrigExpand}[\mathrm{S} / . \backslash[\mathrm{Alpha}]->2 \mathrm{ArcTan}[\backslash[\mathrm{Tau}] / 2]]]
$$

führt zunächst auf die Gleichung

$$
S_{t}(\tau, T 1)=\frac{4 A \tau\left(e^{\frac{T_{R}}{T 1}}-1\right)}{\left(\tau^{2}+4\right) e^{\frac{T_{R}}{T 1}}+\tau^{2}-4},
$$

\footnotetext{
${ }^{3}$ Die beiden neuen Größen sind hier die Zeit zwischen dem anregenden Puls und dem gemessenen Signal (Echozeit $T_{E}$ ) und die effektive Querrelaxationszeit $T_{2}^{*}<T_{2}$. In der Literatur zur Kernspintomografie werden meist die Subscripte weggelassen, und man schreibt $T 1, T 2, T R$ und $T E$. Diese Schreibweise ist auch für Computeralgebra günstig und wird hier nach und nach übernommen.
} 


\section{Anwendungen in der Physik}

welche gegenüber der manuellen Version positive Exponenten besitzt, daher etwas Vorsicht. Mit

Str=FullSimplify [TrigExpand [St

$$
\text { /.Subscript[T, R]->2 T1 ArcTanh[\[Rho]/2]]] }
$$

erhält man dann die rationale Form

$$
S_{t r}(\tau, \rho)=\frac{2 A \rho \tau}{2 \rho+\tau^{2}} .
$$

Diese ist für weitere Computeralgebra günstig.

Eine elementare Kurvendiskussion vereinfacht die Formel weiter. Die Ableitung dieses Signals nach dem Halbtangens $\tau$ lautet

$$
\frac{\partial S_{t r}}{\partial \tau}=\frac{2 A \rho\left(2 \rho-\tau^{2}\right)}{\left(2 \rho+\tau^{2}\right)^{2}}
$$

und hat eine Nullstelle beim Signalmaximum $\tau_{E}^{2}=2 \rho$. An dieser Stelle hat das Signal den Funktionswert

$$
S_{E}=A \sqrt{\rho / 2} \text {. }
$$

Mit der Normierung $s:=S / S_{E}$ und der Skalierung $q:=\tau / \tau_{E}$ gewinnt die rationale Form der ERNST-Gleichung (4.14) schließlich die besonders einfache Form

$$
s=\frac{2 q}{1+q^{2}}=\sin (2 \operatorname{atan}(q)),
$$

vgl. Abb. 4. Insbesondere ist $S(q)=S(1 / q)$ eine Eigenschaft dieser Gleichung.

Die Gleichung (4.T7) offenbart eine fundamentale Symmetrie der ERNSTGleichung. Man erhält die Beziehung

$$
\frac{s}{q}+s q=2
$$

was, wieder ausgedrückt mit den unskalierten Größen, bedeutet

$$
2=\frac{S / S_{E}}{\tau / \tau_{E}}+S / S_{E} \cdot \tau / \tau_{E}=\frac{S / \tau}{S_{E} / \tau_{E}}+\frac{S \tau}{S_{E} \tau_{E}} .
$$




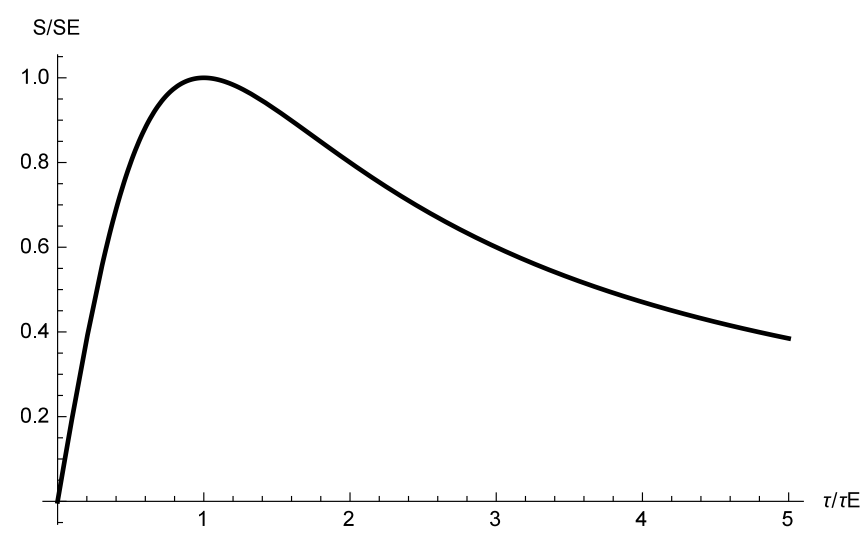

Abbildung 4.1: Die skalierte und normierte ERNST-Gleichung.

Mit den experimentell zugänglichen Größen $y:=S / \tau$ und $x:=S \tau$ ist das eine Geradengleichung

$$
y=\frac{S}{\tau}=2 \frac{S_{E}}{\tau_{E}}-S \tau / \tau_{E}^{2}=A-\frac{x}{2 \rho},
$$

auch direkt mit (4.14) zu verifizieren. Die Koeffizienten $A$ und $\rho$ des untersuchten Gewebevoxels sind also für mehr als zwei Flipwinkel (und damit $\tau)$ mittels einer linearen Regression bestimmbar.

Statt der Größen $S$ und $\tau$ kann man alternativ $x$ und $y$ verwenden, denn die Abbildung $(S, \tau) \leftrightarrow(x, y)$ ist für den physikalisch relevanten Quadranten $S>0$ und $\tau>0$ eine Bijektion: Es gilt $S=\sqrt{x y}$ und $\tau=$ $\sqrt{x / y}$. Daher ist die gefundene Gerade $y(x)$ eine inhärente Eigenschaft der ERNST-Gleichung, und keine Folge der vorgenommenen nichtlinearen Transformation.

\subsection{Die balancierte SSFP-Sequenz}

Die balancierte alternierende SSFP-Sequenz $\mathbf{Z}^{\mathbb{W}}$ ist eine Messtechnik mit einer komplexen Abfolge spezieller Einzelpulse. Ihre Signalstärke hängt

\footnotetext{
${ }^{4}$ Die Abkürzung bSSFP steht für Balanced Steady-State Free Precession. Andere Bezeichnungen sind TrueFISP (Fast Imaging with Steady-state Precession), FIESTA (Fast Imaging Employing STeady-state Acquisition) oder b-FFE (Balanced Fast Field Echo).
} 


\section{Anwendungen in der Physik}

zusätzlich von der Querrelaxation $r 2$ ab. Sie ist gegeben zu

$$
S=\frac{A \sin (\alpha)\left(1-e^{-\mathrm{r} 1 \mathrm{TR}}\right)}{\cos (\alpha)\left(-\left(e^{-\mathrm{r} 1 \mathrm{TR}}-e^{-\mathrm{r} 2 \mathrm{TR}}\right)\right)-e^{-\mathrm{r} 1 \mathrm{TR}-\mathrm{r} 2 \mathrm{TR}}+1},
$$

wobei $r 1=1 / T 1$ und $r 2=1 / T 2$ die Raten der Relaxationsprozesse und $A$ die Amplitude $A \sim M_{0} \exp (-r 2 T R / 2)$ sind, vgl. [SG.J $\left.{ }^{+} 04\right]$. Mit den Ersetzungen

$$
\begin{aligned}
\text { Ers }=\{\backslash[\text { Alpha }] & \rightarrow 2 \operatorname{ArcTan}[\backslash[\mathrm{Tau}] / 2], \\
r 2 & \rightarrow 2 \operatorname{ArcTanh}[\backslash[\mathrm{Rho}] 2 / 2] / \mathrm{TR}, \\
r 1 & \rightarrow 2 \operatorname{ArcTanh}[\backslash[\mathrm{Rho}] 1 / 2] / \mathrm{TR}\}
\end{aligned}
$$

erhält man mit

Srat $=$ FullSimplify $[$ TrigExpand [PowerExpand [S/.Ers $]]$

sofort die rationale Form

$$
S_{\text {rat }}=\frac{A \rho 1(\rho 2+2) \tau}{4 \rho 1+\rho 2 \tau^{2}}
$$

Wieder diskutiert man diese Funktion. Das Maximum liegt bei

$$
\tau_{\max }=2 \sqrt{\frac{\rho 1}{\rho 2}}
$$

und das zugehörige maximale Signal ist

$$
S_{\max }=\frac{1}{4} A(\rho 2+2) \sqrt{\frac{\rho 1}{\rho 2}} .
$$

Normierung auf diese Amplitude und Skalierung auf $\tau_{\max }$ ergibt mit $\mathrm{s}=$ FullSimplify $[($ Srat $/ . \backslash[\mathrm{Tau}] \rightarrow \mathrm{q} \backslash[\mathrm{Tau}] \max ) /$
$\quad \operatorname{Smax},\{\backslash[\mathrm{Rho}] 1>0, \backslash[\mathrm{Rho}] 2>0, \backslash[\mathrm{Tau}]>0\}]$

wieder die besonders einfache Form (4.T)

$$
s=\frac{2 q}{q^{2}+1},
$$

diesmal mit der linearen Beziehung

$$
4 S / \tau+\frac{\rho 2}{\rho 1} S \tau=A(2+\rho 2) .
$$

Die True-Fisp-Sequenz erlaubt also nur die Bestimmung des Quotienten $\rho 2 / \rho 1$ (zusammen mit der mit $\rho 2$ vermengten Amplitude). Dies ist die tiefere Bedeutung der Behauptung, die Sequenz sei „T1/T2-gewichtet“. 


\subsection{Die unbalancierte SSFP-Sequenz}

Diese Sequenz von HF-Pulsen ist eine sehr allgemeine Messtechnik der magnetischen Kernresonanz. Ich folge hier den Herleitungen von GYNGELL [Gyn89] sowie HÄNICKE und VOGEL [HV03].

In der Literatur zur Kernspintomografie werden statt $T 1$ und $T 2$ gerne die Abkürzungen

$$
\begin{aligned}
& E 1=\exp \left(-T_{R} / T 1\right)=\exp \left(-r 1 T_{R}\right) \quad \text { und } \\
& E 2=\exp \left(-T_{R} / T 2\right)=\exp \left(-r 2 T_{R}\right)
\end{aligned}
$$

verwandt. Aus den Gleichungen (ㅍ.3.3) erhält man direkt die Ersetzungen

$$
\begin{aligned}
\text { Ers }=\{\backslash[\text { Alpha }] & >2 \operatorname{ArcTan}[\backslash[\mathrm{Tau}] / 2], \\
\text { E1 } & \rightarrow(2-\backslash[\mathrm{Rho}] 1) /(2+\backslash[\mathrm{Rho}] 1), \\
\text { E2 } & \rightarrow(2-\backslash[\mathrm{Rho}] 2) /(2+\backslash[\mathrm{Rho}] 2)\}
\end{aligned}
$$

für Mathematica. Die physikalisch sinnvollen Beschränkungen der Parameter lauten wegen $0<E_{1,2}<1$. Wieder in Mathematica übersetzt lauten diese mathematischen Annahmen

$$
\begin{aligned}
\text { Ass }=\{\backslash[\mathrm{Tau}]>0, & \backslash[\mathrm{Rho}] 1>0, \backslash[\mathrm{Rho}] 1<2, \\
& \backslash\left[\mathrm{Rho} 02^{>}>0, \backslash[\mathrm{Rho}] 2<2\right\}
\end{aligned}
$$

Es gilt nun, die unbekannten Koeffizienten $p, u$ und $v$ in den beiden Signalen

$$
\begin{aligned}
S_{\mathrm{FID}} & =\frac{A(1-E 1)}{p} \sin (\alpha)(u-E 2 v) \\
S_{\mathrm{Echo}} & =\frac{A(1-E 1)}{p} \sin (\alpha)\left(E 2^{2} u-E 2 v\right)
\end{aligned}
$$

zu bestimmen. Hier werden mit einer Sequenz tatsächlich zwei Signale bestimmt.

Aus den beiden Definitionen

$$
\begin{aligned}
p & =-\mathrm{E} 1 \cos (\alpha)+\mathrm{E} 2^{2}(-(\mathrm{E} 1-\cos (\alpha)))+1 \\
q & =(1-\mathrm{E} 1) \mathrm{E} 2(\cos (\alpha)+1)
\end{aligned}
$$

berechnet man mit den obigen Ersetzungen und Annahmen die Größe

$$
r=\frac{q}{2 p}=-\frac{\rho 1\left(\rho 2^{2}-4\right)}{2 \rho 1\left(\rho 2^{2}+4\right)+4 \rho 2 \tau^{2}} .
$$

Weiter berechnet man mit Computeralgebra die symbolischen Summen 
4 Anwendungen in der Physik

$\mathrm{u}=1+\operatorname{Sum}\left[\mathrm{r}^{\wedge}(2 \mathrm{~m}) \operatorname{Binomial}[2 \mathrm{~m}, \mathrm{~m}],\{\mathrm{m}, 1, \backslash[\right.$ Infinity $\left.]\}\right]$

$\mathrm{v}=\operatorname{Sum}\left[\mathrm{r}^{\wedge}(2 \mathrm{~m}-1)\right.$ Binomial $[2 \mathrm{~m}, \mathrm{~m}],\{\mathrm{m}, 1, \backslash[$ Infinity $\left.]\}\right] / 2$

und vereinfacht diese zu

$$
\begin{aligned}
& u=\frac{\rho 1\left(\rho 2^{2}+4\right)+2 \rho 2 \tau^{2}}{2 w} \\
& v=\frac{\left(\rho 1\left(\rho 2^{2}+4\right)+2 \rho 2 \tau^{2}\right)\left(-\rho 1\left(\rho 2^{2}+4\right)-2 \rho 2 \tau^{2}+2 w\right)}{2 \rho 1\left(\rho 2^{2}-4\right) w}
\end{aligned}
$$

mit der Abkürzung $w$ für die in beiden Summen vorkommende Wurzel

$$
w:=\sqrt{\rho 2\left(\rho 1 \rho 2+\tau^{2}\right)\left(4 \rho 1+\rho 2 \tau^{2}\right)}
$$

Damit ergeben sich die beiden Signalgleichungen

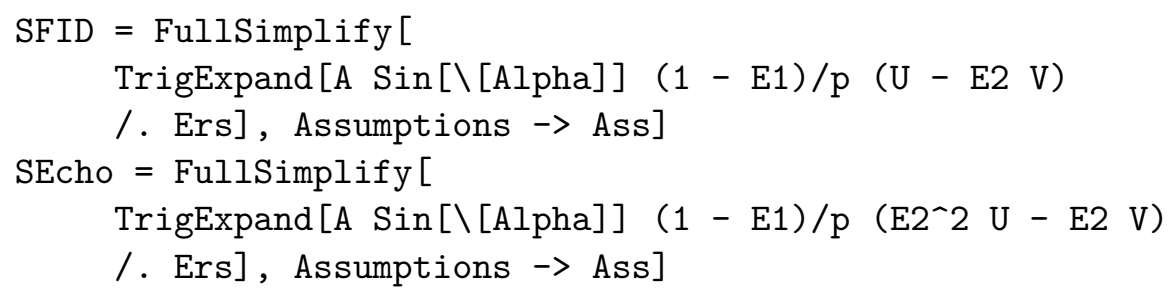

nach Substitution von $w$ und weiteren Vereinfachungen $\mathrm{zu}$

$$
\begin{aligned}
S_{\mathrm{FID}} & =\frac{1}{2} \mathrm{~A} \tau\left(1+\frac{w\left(2 \rho 1-\tau^{2}\right)}{\left(\rho 1 \rho 2+\tau^{2}\right)\left(4 \rho 1+\rho 2 \tau^{2}\right)}\right) \\
S_{\text {Echo }} & =\frac{1}{2} \mathrm{~A} \tau\left(1-\frac{w\left(2 \rho 1+\tau^{2}\right)}{\left(\rho 1 \rho 2+\tau^{2}\right)\left(4 \rho 1+\rho 2 \tau^{2}\right)}\right)
\end{aligned}
$$

Streng genommen sind die Amplituden beider Signale leicht unterschiedlich, da sie nacheinander aufgenommenen werden. Insgesamt ist interessant, dass durch die unendliche Summation der Effekte der Einzelimpulse (alle beschrieben durch rationale Funktionen) ein Wurzelterm entsteht, welcher keiner rationale Funktion mehr ist.

Beide Signale enthalten dieselbe Wurzel zusammen mit einem identischen Nenner. Nach diesem Term löst man beide Signale auf und setzt diese gleich. So erhält man die Beziehung

$$
\frac{S_{\text {Echo }}+S_{\mathrm{FID}}}{\tau}=\mathrm{A}+\frac{\left(S_{\mathrm{Echo}}-S_{\mathrm{FID}}\right) \tau}{2 \rho 1} .
$$


Wieder kann man durch eine geschickte lineare Auftragung die Parameter $\rho 1$ und $A$ bestimmen. Den noch fehlenden Parameter $\rho 2$ bestimmt man etwa aus der Differenz $d(\tau):=S_{\text {FID }}-S_{\text {Echo }}$ über die Beziehung

$$
2 \frac{\rho 1 A^{2}}{d(\tau)^{2}}-\frac{2 \rho 1}{\tau^{2}}-\frac{\tau^{2}}{2 \rho 1}=\frac{\rho 2}{2}+\frac{2}{\rho 2} .
$$

Man erhält eine quadratische Gleichung, deren Lösungen

$$
\begin{aligned}
\rho 2= & \frac{1}{2 d^{2} \rho 1 \tau^{2}}\left(4 \mathrm{~A}^{2} \rho 1^{2} \tau^{2}-d^{2}\left(4 \rho 1^{2}+\tau^{4}\right)\right. \\
& \pm \sqrt{\left.\left(4 \mathrm{~A}^{2} \rho 1^{2} \tau^{2}-d^{2}\left(4 \rho 1^{2}+\tau^{4}\right)\right)^{2}-16 d^{4} \rho 1^{2} \tau^{4}\right)}
\end{aligned}
$$

beide positiv sind, denn nach Einsetzen der Signalgleichungen gilt

$$
4 \mathrm{~A}^{2} \rho 1^{2} \tau^{2}-d^{2}\left(4 \rho 1^{2}+\tau^{4}\right)=\frac{4 \mathrm{~A}^{2} \rho 1^{3}\left(\rho 2^{2}+4\right) \tau^{4}}{\left(\rho 1 \rho 2+\tau^{2}\right)\left(4 \rho 1+\rho 2 \tau^{2}\right)}>0 .
$$

Aber nur die Lösung mit „- " liefert die gewünschten kleine Werte für $\rho 2$.

\subsection{Sequenzen aus Einzelpulsen}

Kann man die Effekte eines einzelnen Pulses mathematisch beschreiben, so ist auch eine Sequenz von Pulsen beschreibbar. Genau das ist der Ansatz von Woessner [Woe61], weshalb die die Pulse beschreibenden Matrizen Woessner-Propagatoren genannt werden.

Die komplexe Magnetisierung $m(t)=m_{x}(t)+i m_{y}(t)$ und die $z$-Komponente $m_{z}$ sind Funktionen der Zeit. Sie können daher in eine komplexe Fourierreihe entwickelt werden. In der Arbeit von HENNIG et al. [HWS(04], der ich folge, wird dies formalisiert. Diese Arbeit fußt auf der genannten Arbeit von WoEsSNer.

Mit den Koeffizienten

$$
m_{x}+i m_{y}=\sum_{k=-\infty}^{\infty} F_{k} e^{i k \omega} \text { und } m_{z}=\sum_{k=-\infty}^{\infty} Z_{k} e^{i k \omega}
$$

kann die Wirkung eines Einzelpulses auf die $k$-ten Fourierkoeffizienten $\left(F_{k}, F_{-k}, Z_{k}\right)$ beschrieben werden durch die Matrix

$$
\boldsymbol{T}(k, \alpha)=\left(\begin{array}{ccc}
\cos ^{2} \frac{\alpha}{2} & \sin ^{2} \frac{\alpha}{2} & -\sin \alpha \\
\sin ^{2} \frac{\alpha}{2} & \cos ^{2} \frac{\alpha}{2} & \sin \alpha \\
-\frac{1}{2} \sin \alpha & \frac{1}{2} \sin \alpha & \cos \alpha
\end{array}\right)
$$




\section{Anwendungen in der Physik}

Diese ist algebraisierbar, denn es gilt unter Benutzung von (ㄸ. (ㅁ. 2)

$$
\begin{aligned}
\boldsymbol{T}(k, \alpha) & =\left(\begin{array}{ccc}
\frac{1}{1+t^{2}} & \frac{t^{2}}{1+t^{2}} & -\frac{2 t}{1+t^{2}} \\
\frac{t^{2}}{1+t^{2}} & \frac{1}{1+t^{2}} & \frac{2 t}{1+t^{2}} \\
-\frac{1}{2} \frac{2 t}{1+t^{2}} & \frac{1}{2} \frac{2 t}{1+t^{2}} & \frac{1-t^{2}}{1+t^{2}}
\end{array}\right) \\
& =\frac{1}{1+t^{2}}\left(\begin{array}{ccc}
1 & t^{2} & -2 t \\
t^{2} & 1 & 2 t \\
-t & t & 1-t^{2}
\end{array}\right) .
\end{aligned}
$$

Die Relaxationseffekte zwischen Pulsen und Echo werden einfach durch die Diagonalmatrix $\boldsymbol{D}\left(T_{E} / 2\right)$ und ihre Exponentialabbildung beschrieben. Mit

$$
\begin{aligned}
\exp \left(\boldsymbol{D}\left(\frac{T_{E}}{2}\right)\right) & =\left(\begin{array}{ccc}
\exp \left(-\frac{T_{E}}{2 T_{2}}\right) & 0 & 0 \\
0 & \exp \left(-\frac{T_{E}}{2 T_{2}}\right) & 0 \\
0 & 0 & \exp \left(-\frac{T_{E}}{2 T_{1}}\right)
\end{array}\right) \\
& =\left(\begin{array}{ccc}
\sqrt{E_{2}} & 0 & 0 \\
0 & \sqrt{E_{2}} & 0 \\
0 & 0 & \sqrt{E_{1}}
\end{array}\right)
\end{aligned}
$$

erhält man mit der hyperbolischen Ersetzung im Vierteltangens

$$
\begin{aligned}
& \mathrm{E} 1 / . \mathrm{T} 1 \rightarrow \mathrm{TE} /(4 \operatorname{ArcTanh}[\mathrm{rho} / 4]) \\
& \mathrm{E} 2 / . \mathrm{T} 2 \rightarrow \mathrm{TE} /(4 \operatorname{ArcTanh}[\mathrm{rho} / 4])
\end{aligned}
$$

die Algebraisierung

$$
\exp \left(\boldsymbol{D}\left(\frac{T_{E}}{2}\right)\right)=\left(\begin{array}{ccc}
\frac{4-\rho 2}{4+\rho 2} & 0 & 0 \\
0 & \frac{4-\rho 2}{4+\rho 2} & 0 \\
0 & 0 & \frac{4-\rho 1}{4+\rho 1}
\end{array}\right) .
$$

Da diese Matrizen aber meist quadratisch auftauchen, reicht oft eine Substitution im halben Argument. Damit machen die Halbwinkelsubstitution und ihr (höheres) hyperbolisches Analogon auch Sinn in der Beschreibung von Einzelpulsen.

Wieder Hennig beschreibt in [Hen91] unter Berufung auf Woessner 
[Woe61] die Wirkung einer HF-Pulssseqenz mit Hilfe der Gesamtmatrix

$$
T_{p}=\left(\begin{array}{ccccc}
T_{0} & & & & \\
& T_{1} & & & \\
& & T_{1} & & \\
& & & T_{1} & \\
& & & & \ddots
\end{array}\right),
$$

wobei die Einzelpulse durch die Matrizen

$$
T_{0}=\left(\begin{array}{ccc}
1 & & \\
& \cos \alpha & -\sin \alpha \\
& \sin \alpha & \cos \alpha
\end{array}\right)
$$

und

$$
T_{1}=\left(\begin{array}{cccc}
\cos ^{2} \frac{\alpha}{2} & \sin ^{2} \frac{\alpha}{2} & \sin \alpha & 0 \\
\sin ^{2} \frac{\alpha}{2} & \cos ^{2} \frac{\alpha}{2} & 0 & \sin \alpha \\
\frac{1}{2} \sin \alpha & -\frac{1}{2} \sin \alpha & \cos \alpha & 0 \\
-\frac{1}{2} \sin \alpha & \frac{1}{2} \sin \alpha & 0 & \cos \alpha
\end{array}\right)
$$

beschrieben werden, welche beide algebraisierbar sind. Es ergibt sich nämlich wie gehabt

$$
T_{0}=\left(\begin{array}{ccc}
1 & & \\
& \frac{1-t^{2}}{1+t^{2}} & -\frac{2 t}{1+t^{2}} \\
& \frac{2 t}{1+t^{2}} & \frac{1-t^{2}}{1+t^{2}}
\end{array}\right)=\frac{1}{1+t^{2}}\left(\begin{array}{ccc}
1+t^{2} & & \\
& 1-t^{2} & -2 t \\
& 2 t & 1-t^{2}
\end{array}\right)
$$

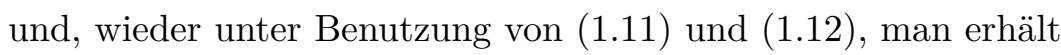

$$
\begin{aligned}
T_{1} & =\left(\begin{array}{cccc}
\frac{1}{1+t^{2}} & \frac{t^{2}}{1+t^{2}} & \frac{2 t}{1+t^{2}} & 0 \\
\frac{t^{2}}{1+t^{2}} & \frac{1}{1+t^{2}} & 0 & \frac{2 t}{1+t^{2}} \\
\frac{1}{2} \frac{2 t}{1+t^{2}} & -\frac{1}{2} \frac{2 t}{1+t^{2}} & \frac{1-t^{2}}{1+t^{2}} & 0 \\
-\frac{1}{2} \frac{2 t}{1+t^{2}} & \frac{1}{2} \frac{2 t}{1+t^{2}} & 0 & \frac{1-t^{2}}{1+t^{2}}
\end{array}\right) \\
& =\frac{1}{1+t^{2}}\left(\begin{array}{cccc}
1 & t^{2} & 2 t & 0 \\
t^{2} & 1 & 0 & 2 t \\
t & -t & 1-t^{2} & 0 \\
-t & t & 0 & 1-t^{2}
\end{array}\right) .
\end{aligned}
$$

Damit ist die Gesamtmatrix $T_{p}$ algebraisierbar; bei gleichen Flipwinkeln der Einzelpulse sogar mit einem gemeinsamen Vorfaktor $\frac{1}{1+t^{2}}=\cos ^{2}\left(\frac{\alpha}{2}\right)$ 
4 Anwendungen in der Physik

(zur entsprechenden Potenz erhoben). Dann sind recht einfache Formen für die damit berechenbaren Signalgleichungen zu erwarten.

In der Grenze, also für unendlichdimensionale $T_{p}$, ergeben sich aber wieder ggf. nicht-algebraische Funktionen. 


\section{Literaturverzeichnis}

[Blo46] Bloch, Felix: Nuclear induction. In: Physical review 70 (1946), Nr. 7-8, 460. http://journals.aps.org/pr/ abstract/10.1103/PhysRev.70.460

[Dar14] Darboux: Theorie des surfaces. 2. Ed. Paris, 1914

[DH10] Dathe, Henning ; Helms, Gunther: Exact algebraization of the signal equation of spoiled gradient echo MRI. In: Physics In Medicine and Biology 55 (2010), August, Nr. 15, S. 42314245. http://dx.doi.org/10.1088/0031-9155/55/15/003. - DOI 10.1088/0031-9155/55/15/003

[Fre54] Freudenstein, F.: An Analytical Approach to the Design of Four-Link Mechanisms. In: ASME Trans 76(3) (1954), S. 483-492

[FVJH57] Feynman, Richard P. ; Vernon JR, Frank L. ; Hellwarth, Robert W.: Geometrical representation of the Schrödinger equation for solving maser problems. In: Journal of applied physics 28 (1957), Nr. 1, 49-52. http://scitation.aip. org/content/aip/journal/jap/28/1/10.1063/1.1722572

[Gan58] GantmacheR, FR.: Matrizenrechnung I. VEB Deutscher Verlag der Wissenschaften, 1958

[Gyn89] Gyngell, M. L.: An analytical solution for the SSFP signal in MRI. In: Journal of Magnetic Resonance 81 (1989), S. $474-483$

[HD64] Hartenberg, R.S. ; Denavit, J.: Kinematic Synthesis of Linkages. McGraw-Hill, 1964

[HDD08] Helms, Gunther ; Dathe, Henning ; Dechent, Peter: Quantitative FLASH MRI at 3T using a rational approximation of the Ernst equation. In: Magnetic Resonance In Medicine 
Literaturverzeichnis

59 (2008), März, Nr. 3, S. 667-672. http://dx.doi.org/10. 1002/mrm.21542. - DOI 10.1002/mrm.21542

[HDWD11] Helms, Gunther ; Dathe, Henning ; Weiskopf, Nikolaus ; Dechent, Peter: Identification of Signal Bias in the Variable Flip Angle Method by Linear Display of the Algebraic Ernst Equation. In: Magnetic Resonance In Medicine 66 (2011), September, Nr. 3, S. 669-677. http://dx.doi.org/ 10.1002/mrm.22849. - DOI 10.1002/mrm.22849

[Hen91] Hennig, J.: Echoes -How to Generate, Recognize, Use or Avoid Them in MR-Imaging Sequences. In: Concepts in Magnetic Resonance 3 (1991), S. 125-143

[HFM $\left.{ }^{+} 85\right]$ Haase, A ; Frahm, J ; Matthei, D ; Hänicke, W ; MerBOLDT, KD: FLASH Imaging. Rapid NMR Imaging Using Low Flip-Angle Pulses. In: Journal of Magnetic Resonance 67 (1985), April, Nr. 2, S. 258-266. - DOI: 10.1016/00222364(86)90433-6

[HV03] HäNicke, Wolfgang ; Vogel, Horst U.: An analytical solution for the SSFP signal in MRI. In: Magn Reson Med 49 (2003), Apr, Nr. 4, 771-775. http://dx.doi.org/10.1002/ mrm.10410. - DOI 10.1002/mrm.10410

[HWS04] Hennig, Juergen ; Weigel, Matthias ; Scheffler, Klaus: Calculation of flip angles for echo trains with predefined amplitudes with the extended phase graph (EPG)-algorithm: Principles and applications to hyperecho and TRAPS sequences. In: Magnetic resonance in medicine 51 (2004), Nr. 1, 68-80. http://onlinelibrary.wiley.com/doi/10.1002/ mrm.10658/full

[JR94] Jeffrey, D.J. ; Rich, A.D.: The Evaluation of Trigonometric Integrals Avoiding Spurious Discontinuities. In: $A C M$ Transactions on Mathematical Software 20 (1994), Nr. 1, S. 124-135

[Kam77] Kamke, Erich: Differentialgleichungen - Lösungsmethoden und Lösungen. B. G. Teubner, 1977

[LM90] Luck, K. ; Modler, KH.: Getriebetechnik. Springer, 1990 
[Mül59] MüLlER, Hans R.: Zur Ermittlung von Hüllflächen in der räumlichen Kinematik. In: Monatshefte für Mathematik 63 (1959), S. 231-240

[Rem92] Remmert, R.: Funktionentheorie 1. 3. Auflage. Springer, 1992

[SGJ $\left.{ }^{+} 04\right]$ Schmitt, Peter ; Griswold, Mark A. ; Jakob, Peter M. ; Kotas, Markus ; Gulani, Vikas ; Flentue, Michael ; HaASE, Axel: Inversion recovery TrueFISP: quantification of T(1), $\mathrm{T}(2)$, and spin density. In: Magn Reson Med 51 (2004), Apr, Nr. 4, 661-667. http://dx.doi.org/10.1002/mrm.20058. DOI $10.1002 / \mathrm{mrm} .20058$

[SH97] Siminovitch, DJ ; Habot, S: An NMR rotation operator disentanglement strategy for establishing properties of the Euler-Rodrigues parameters. In: Journal of Physics A - Mathematical and General 30 (1997), APR 7, Nr. 7, S. 2577-2584. http://dx.doi.org/10.1088/0305-4470/30/7/033. - DOI 10.1088/0305-4470/30/7/033. - ISSN 0305-4470

[Shu93] Shuster, MD.: A Survey of Attitude Representations. In: Journal of the Astronautical Sciences 41 (1993), OCT-DEC, Nr. 4, S. 439-517. - ISSN 0021-9142

[SJH84] Silver, MS ; Joseph, RI ; Hoult, DI: Highly selective $\pi / 2$ and $\pi$ pulse generation. In: Journal of Magnetic Resonance (1969) 59 (1984), Nr. 2, 347351. http://www.sciencedirect.com/science/article/ pii/0022236484901811

[Spi80] SpIVAK, Michael: Calculus. 2nd Ed. Publish or Perish, 1980

[Stu64] Stuelpnagel, JC.: On the Parametrisation of the ThreeDimensional Rotation Group. In: SIAM Review 6 (1964), S. $422-430$

[Wal90] WaLter, Wolfgang: Gewöhnliche Differentialgleichungen. 4. Auflage. Springer-Verlag, 1990

[Woe61] Woessner, D.E.: Effects of Diffusion in Nuclear Magnetic Resonance Spin-Echo Experiments. In: Journal of Chemical Physics 34 (1961), S. 6 



\section{Personen- und Sachverzeichnis}

BLOCH-RICCATI, 60]

BLOCH, 55, 57

BoHR, 56]

Boltzmann, 56

Bose-Einstein, 5.

Cayley-Klein, 而

CAyley, 401, 47, 48, [3.3

Darboux, B7, 50

EISENSTEIN, 35

ERNST, [2], 63]

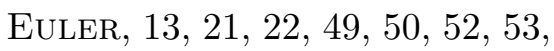

5.5

FERMAT, [3]

FERMI-DiRAC, 55

FEYNMAN, 57

Freudenstein, t.3.

Gauss, [2.:

GyNGELL, 6.5

HÄNICKE, 65

HAASE, [1]

HeLms, 8

Hennig, 67, 68

JEFFREY, 2]

KAMKE, $\mathbf{3 Z}$

LANDÉ, 56

LARMOR, 58

MöBIUs, पᄄ4

MÜLLER, 38

MaXwell-Boltzmann, 5.5

Moivre, [2.5, [26], 5]

PAULI, 可
Pythagoras, ㅁ]

Riccati, 8, 56, 38, 52

Rich, 2]

Rodrigues-CAyley, 四

Rodrigues, 4.5 -4.1, 5.3

SILVER, B87, 5.9]

Siminovitch, 38

SPIVAK, [

VOGEL, 65

WEIERSTRASS, ש]

WOESSNER, [7, [78

Additionstheorem, 国, 331, 40

Bahn, 52

Besetzungszahldifferenz, 囵

Bloch-Gleichung, 57

bSSFP, 63

Cayley-Klein-Darstellung, 每

Cayley-Parameter, 40, 47, 48, 50]

Computeralgebra, [0]

Drehungen, 3.9, 45

Einheitswurzeln, [27, [2]

Eisenstein-Funktion, 35

Eulerparameter, 48, 4.91

Eulersche Formel, 미

FFE, 60

FIESTA, 6.3 
Personen- und Sachverzeichnis

FLASH, 60]

höhere Vielfache, [25, 42, [68

Hüllflächen, $\mathbf{3 8}$

Halbwinkelsatz, 2.3

Kinemate, 52

Kinematik, 52

kinematische Invariante, 52

kleine Argumente, 미, [5]

komplex, [13], 지, [28, [1], 34, 37, 5], 50, 67

Kreuzprodukt, 45, 48

Kugelkoordinaten, 32

Merkregel, ㅁ]

Pauli-Matrizen, 50

Polarkoordinaten, 30

Pythagoreisches Zahlentripel, [2.3

Quaternionen, 50

rationale Funktionen, 20

Riccati-Differentialgleichung, [56,

[38, 52, 57, 5.9, 50

Rodrigues-Parameter, 40, 46, 40,

50

Schraubparameter, 5.3

Schraubung, 5.3

SPGR, 60]

TrueFISP, 63

Verteilungsfunktionen, 5.5

Viergelenk, 43

Woessner-Propagatoren, 67 


\section{Abbildungsverzeichnis}

1.1 Die Funktionen $\sin \alpha=\frac{2 t}{1+t^{2}}$ und $\cos \alpha=\frac{1-t^{2}}{1+t^{2}}$ als Funktion
des Tangens des halben Winkels. Der Definitionsbereich
$\alpha \in(-\pi, \pi)$ wird abgebildet auf $t \in(-\infty, \infty)$. Genauer
bekommt man für jeden Vollwinkelbereich $\alpha \neq n \pi$, wobei
n eine ganze Zahl ist, wieder den gesamten Bereich für $t$.
Der Wertebereich bleibt natürlich ungeändert. . . . . . .

1.2 Die Merkregel zur Halbwinkelsubstitution. . . . . . . . . . 11

1.3 Die Visualisierung der Halbwinkelsubstitution. Weitere EinEelheiten im Text des Abschnittes 1.3 . . . . . . . . . 12

1.4 Die Funktionen $\sinh x=\frac{2 h}{1-h^{2}}$ und $\cosh x=\frac{1+h^{2}}{1-h^{2}}$ sowie $\exp x=\frac{1+h}{1-h}$ als Funktion des Tangenshyperbolikus des halben Arguments. . . . . . . . . . . . . . .

2.1 Das erste Auftreten der Halbwinkelsubstitution bei EULER

$2.2 \quad$ Das Einheitsdreieck mit den Standardbeschriftungen. . . . 24

2.3 Die fünften Einheitswurzeln. Sei $\alpha=\frac{360^{\circ}}{n}$ der Innenwinkel des regelmäßigen $n$-Ecks. Hat die Strecke $s=\sin \frac{\alpha}{2}$ eine mit Zirkel und Lineal konstruierbare Länge, so ist genau dann das regelmäßjige n-Eck mit Zirkel und Lineal konstruierbar. Denn: Schlage einen Kreis $k$ mit Radius $s$ um $B$ und konstruiere die Tangente von $A$ an $k$. Die Strecke $s$ steht senkrecht auf dieser Tangente. Verdoppele $s$ und wiederhole die gesamte Konstruktion um den Punkt $B^{\prime}$. . 28

2.4 Die Halbwinkelsubstitution als stereografische Projektion.

$2.5 \quad$ Der Arcustangens von einem $\arctan (y / x)(2.30)$ und zwei Argumenten $\arctan (x, y)(2.31)$ in Mathematica. In manchen Programmiersprachen ist die Reihenfolge der Argumente vertauscht . . . . . . . . . . . . . . . 32 
Abbildungsverzeichnis

3.1 Ein Viergelenk, hier eine Kurbelschwinge. Die Strecke $\overline{A D}$ wird als befestigt angenommen ("Gestell"), die Strecke $A B$ dient typischerweise als Antrieb („Pleuel“) und die Strecke $\overline{D C}$ als Abtrieb. Das bewegte System ist mit der Strecke $B C$ (,Koppel“) verbunden. Die Winkel werden relativ zur Geraden $d_{0}$ gemessen . . . . . . . . . . . . . 43

4.1 Die skalierte und normierte ERNST-Gleichung. . . . . . . 63 

Das vorliegende Büchlein wendet sich alle, die sich für Rechentricks interessieren. Der hier behandelte Trick ist die sog. Halbwinkelsubstitution, welche erheblich mehr Anwendungen hat, als die Anfängervorlesung vermuten lässt. Ab etwa dem dritten oder vierten Semester sollten die präsentierten Beispiele für Physiker, Mathematiker oder Ingenieure verständlich sein.

Nach einer elementaren Herleitung der Substitution und der Vorstellung der nötigen mathematischen Werkzeuge folgen Kapitel über beispielhafte Anwendungen in Mathematik, Kinematik und Physik. Letztere entstammen vorwiegend der Kernspintomografie; der Leser möge sich nicht durch den dort verwandten Jargon abschrecken lassen. Eine Signalgleichung etwa ist auch nur eine Gleichung im mathematischen Sinne. Der mit der Kernspintomografie vertraute Leser kann aus den letzten Kapiteln einen vermehrten Nutzen ziehen.

Der tiefere Zusammenhang zwischen den Kapiteln offenbart sich in der Ähnlichkeit der Euler-Gleichungen des starren Körpers mit der Bloch-Gleichung der Kernspinresonanz. Bei beiden ist zur Vereinfachung eine stereografische Projektion hilfreich, welche mit der Halbwinkelsubstitution eng verwandt ist. 